\title{
UNIFORMIZATION OF DELIGNE-MUMFORD CURVES
}

\author{
KAI BEHREND AND BEHRANG NOOHI
}

\begin{abstract}
We compute the fundamental groups of non-singular analytic DeligneMumford curves, classify the simply connected ones, and classify analytic Deligne-Mumford curves by their uniformization type. As a result, we find an explicit presentation of an arbitrary Deligne-Mumford curve as a quotient stack. Along the way, we compute the automorphism 2-groups of weighted projective stacks $\mathcal{P}\left(n_{1}, n_{2}, \cdots, n_{r}\right)$. We also discuss connections with the theory of $F$-groups, 2-groups, and Bass-Serre theory of graphs of groups.
\end{abstract}

\section{INTRODUCTION}

The aim of this paper is to extend the uniformization of Riemann surfaces to Deligne-Mumford analytic curves.

In the case of Riemann surfaces, the uniformization problem is well-understood. There are three simply connected 1-dimensional analytic domains, namely $\mathbb{H}, \mathbb{C}$ and $\mathbb{P}^{1}$. Accordingly, there are three types of analytic curves: hyperbolic, Euclidian, and spherical. The only spherical curve is the Riemann sphere $\mathbb{P}^{1}$. The Euclidian ones are the ones whose universal cover is $\mathbb{C}$. These are precisely $\mathbb{C}, \mathbb{C}^{*}$, and elliptic curves. Everything else has universal cover $\mathbb{H}$, so is hyperbolic.

Every Riemann surface arises as a quotient of one of these simply connected domains by a discrete subgroup of the group of its analytic automorphisms. Such discrete subgroups, being the fundamental groups of the corresponding Riemann surfaces, have a particularly simple form, and they act without fixed points on the corresponding symmetric domain.

In passage to Deligne-Mumford curves, we face some complications, as there are more simply connected Deligne-Mumford curves which were not present in the classical case. For instance, every weighted projective line $\mathcal{P}(m, n)$ is simply connected. The good news is that these are all.

Theorem 1.1. The simply connected Deligne-Mumford curves are precisely $\mathbb{H}, \mathbb{C}$ and $\mathcal{P}(m, n)$, for arbitrary $n, m \geq 1$.

So, the trichotomy hyperbolic/Euclidian/spherical continues to hold in the more general Deligne-Mumford setting: every Deligne-Mumford curve has a universal cover which is either $\mathbb{H}, \mathbb{C}$ or $\mathcal{P}(m, n)$, for some $n, m \geq 1$.

The homotopy groups of a Deligne-Mumford curve are rather easy to compute. It is also easy to determine the uniformization type of a Deligne-Mumford curve by just "looking at it"! (Propositions 7.4, 5.11.)

The next task is to represent a Deligne-Mumford curve as a quotient of its universal cover. This is pretty easy in the hyperbolic and Euclidian cases (Proposition 7.5). The spherical case, however, poses some challenge. The key difference is that, in the spherical case the universal cover is itself a stack, so its automorphisms from a 2-group. This means, to be able to talk about deck transformations, we 
have to incorporate some 2-group theory. As a result the classification of spherical Deligne-Mumford curves is less trivial. The main inputs are: 1) computation of the automorphism 2-groups of weighted projective lines, 2) homotopy classification of maps from discrete groups into these 2-groups. After accomplishing this, we arrive at the following classification results.

Theorem 1.2. Let $m \neq n$ be given positive integer numbers, and let $d=\operatorname{gcd}(m, n)$. Then, the isomorphism classes of Deligne-Mumford curves $\mathcal{X}$ with universal cover $\mathcal{P}(m, n)$ are in natural bijection with isomorphism classes of pairs $(K, \chi)$, where $K$ is a finite group containing $\mu_{d}$ as a central subgroup and $\chi: K / \mu_{d} \rightarrow \mathbb{C}^{*}$ is a character.

Theorem 1.3. All Deligne-Mumford curves whose universal cover is $\mathcal{P}(d, d)$ are given by triples $(\iota, E, \rho)$, where

i. $\iota: \mathbb{C}^{*} \hookrightarrow E$ is a central extension of algebraic groups,

ii. $\rho: E \rightarrow G L_{2}$ is a 2-dimensional representation,

such that the following two conditions are satisfied:

a. $\rho \circ \iota: \mathbb{C}^{*} \rightarrow G L_{2}$ is the diagonal $(d, d)$-th power map,

b. $E / \mathbb{C}^{*}$ is finite.

The above results are constructive. We show in Section 9 how given data as in above theorems one can produce the corresponding Deligne-Mumford curve as a quotient stack. In particular, this gives a canonical presentation of a spherical Deligne-Mumford curve as a quotient stack. (We have such a presentation in the hyperbolic and Euclidian case too.)

The paper is organized as follow. In Section 2 we fix some notations. In Section 3 we overview the theory of analytic stacks. Section 4 serves as a toolbox throughout the paper. In it we collect some easy, and perhaps well-known, facts which will be invoked in the course of proofs or constructions in the ensuing sections. This is to avoid cluttering up the arguments with trivialities.

Section 5 discusses the uniformization problem in the orbifold case. We compute (using van Kampen theorem) the fundamental groups of all orbifold curves, classify the simply connected ones, and classify the orbifold curves by their uniformization type. In Section 7 we extend this to all smooth Deligne-Mumford curves. In this case we have a new family of simply connected stacks which should be taken into consideration. These are the weighted projective lines. In Subsection 7.1 of Section 7 we give a way of computing the fundamental group of a Deligne-Mumford curve avoiding the theorem of van Kampen. The point is that, an (open) DeligneMumford curve contains a graph of groups [11] as a 1-skeleton that is a deformation retract. In particular the formulas in [11] give an alternative way of computing the fundamental groups.

Section 9 augments Section 7, or more precisely, Proposition 7.6 of Section 7, by giving a more conceptual explanation of the theorem and describing the classification of spherical Deligne-Mumford curves (the ones whose universal cover is a weighted projective line).

Acknowledgments. The second author would like to thank University of British Columbia, Newton Institute, and C.I.R.M-Luminy, where the research for this work done, as well as B. Toen and A. Vistoli for helpful discussions. 


\section{CONTEnTS}

1. Introduction 1

2. Notations and terminology 3

3. Overview of topological and analytic stacks 4

3.1. Topological stacks 4

3.2. Complex analytic stacks 5

3.3. Comparing algebraic, analytic and topological stacks 6

4. Some useful facts $\quad 7$

4.1. Basic facts about fundamental groups $\quad 7$

4.2. Stacky discs and their fundamental groups 8

4.3. Weighted projective lines, footballs, and drops 11

5. Uniformization of Deligne-Mumford curves: the case of an orbifold 12

5.1. Proofs 13

5.2. Digression on group presentations 15

6. Interlude: Gerbes over orbifold weighted projective lines 16

7. Uniformization of Deligne-Mumford curves: the general case 19

7.1. Graphs of groups as skeleta of open Deligne-Mumford curves 21

8. Weighted projective general linear 2-groups 23

8.1. Explicit description of $\operatorname{PGL}(m, n) \quad 26$

9. Classification of spherical Deligne-Mumford curves 28

9.1. Universal cover $\mathcal{P}(m, n), m \neq n \quad 29$

9.2. Universal cover $\mathcal{P}(d, d) \quad 31$

9.3. Conjugacy classes in $\operatorname{Hom}_{\mathrm{Ho}(\mathbf{2 G p})}(\Gamma, \operatorname{PGL}(d, d)) \quad 32$

10. Appendix: 2-groups 34

10.1. Morphisms into 2-groups 36

10.2. Conjugations in 2-groups and crossed-modules 37

$\begin{array}{ll}\text { References } & 38\end{array}$

\section{Notations AND TERminology}

We use the terms Riemann surface and analytic curve interchangeably. All Riemann surfaces and Deligne-Mumford curves considered in this paper are assumed to be of finite type (i.e. compact with a finite number of discs, possibly of zero radius, removed).

The symbols $\mathbb{H}, \mathbb{C}$ and $\mathbb{P}^{1}$ stand for the upper half-plane, the complex plane and the Riemann sphere, respectively. We denote the complex unit disc by $\mathbb{D}$ and its stacky quotient under the rotation action of $\mathbb{Z}_{n}$ by $\mathcal{D}_{n}$. We denote the punctured $\operatorname{disc} \mathbb{D}-\{0\}$ by $\mathcal{D}_{\infty}$ or $\mathbb{D}^{*}$. Similarly, we use the notations $\mathcal{C}_{n}$ for $\left[\mathbb{C} / \mathbb{Z}_{n}\right]$, and $\mathcal{C}_{\infty}$ or $\mathbb{C}^{*}$ for $\mathbb{C}-\{0\}$. The complex plane with two orbifold points of order 2 , say at 0 and 1 , is denoted by $\mathcal{C}_{2,2}$.

All stacks are assumed to be smooth and separated. By an orbifold we mean a (smooth) Deligne-Mumford (topological, analytic or algebraic) stack that is generically a (topological, analytic, algebraic) space. (Some authors call this a reduced orbifold.) By an orbifold point of a stack (which is not necessarily an orbifold) we mean a point at which the inertia group jumps (e.g. origin in $\left.\mathcal{D}_{n}, 2 \leq n<\infty\right)$.

We denote the coarse moduli space (sometime called the "underlying space") of a (topological, analytic or algebraic) stack $X, \mathcal{e}$ etc. by $\mathcal{X}_{\bmod }, \mathcal{C}_{\text {mod }}$ etc.. 
We do not want to worry about 2-isomorphisms so 2-isomorphic morphisms are declared equal, except in Sections 8 and 9. When talking about stacks, we use the terms equivalence and isomorphism interchangeably.

We say that a topological stack is uniformizable, if its universal cover is an honest topological space. We point out that the universal cover of a topological stack always exists as a topological stack. This is proven in [7].

For a subgroup $H$ of a group $G$, we denote its centralizer by $Z_{G}(H)$. We denote the center of $G$ by $Z(G)$.

We denote the general linear group over $\mathbb{C}$ by $\mathrm{GL}_{n}(\mathbb{C})$, or more briefly, $\mathrm{GL}_{n}$. Similarly $\mathrm{PGL}_{n}$ stands for the projective general linear group over $\mathbb{C}$.

Sometime we use the term 'map' instead of 'morphism'. For instance, instead of saying a 'morphism $\Gamma \rightarrow \mathfrak{G}$ of 2 -groups' we say a 'map $\Gamma \rightarrow \mathfrak{G}$ of 2 -groups'.

\section{Overview of topological AND ANALYTiC StaCKS}

We recall the basic definitions/facts about topological and analytic stacks. For more details the reader can consult [7].

3.1. Topological stacks. Let Top be the category of topological spaces. We endow Top with the usual Grothendieck topology (covers are simply topological open covers); so we can talk about (the 2-category of) stacks over Top. By Yoneda's lemma, the category of topological spaces embeds as a full subcategory of the 2category of stacks.

We say a morphism $f: y \rightarrow X$ of stacks over Top is representable, if for any map $X \rightarrow X$ from a topological space $X$ to $X$, the fiber product $Y=X \times x y$ is equivalent to a topological space. Let $\mathbf{P}$ be a property of maps of topological spaces that is stable under base change; for example $\mathbf{P}=$ local homeomorphism, $\mathbf{P}=$ open, $\mathbf{P}=$ finite fibers, $\mathbf{P}=$ discrete fibers, or $\mathbf{P}=$ covering map. We say a representable morphism $f: y \rightarrow X$ of stacks over Top satisfies $\mathbf{P}$ if for any map $X \rightarrow X$ from a topological space $X$ to $X$, the base extension $Y \rightarrow X$ of $f$ satisfies $\mathbf{P}$. A pre-Deligne-Mumford topological stack is a stack for which there exists an epimorphism $p: X \rightarrow X$ from a topological space $X$, such that $p$ is representable by local homeomorphisms.

Sometimes we need to work with properties $\mathbf{P}$ that are only invariant under base extension via local homeomorphism. For example $\mathbf{P}=$ closed. In this case, we can still define the property $\mathbf{P}$ for a morphisms $f: y \rightarrow X$ of stacks, provided $X$ is a pre-Deligne-Mumford stack. More precisely, we say that $f: y \rightarrow X$ satisfies $\mathbf{P}$, if for any local homeomorphism $X \rightarrow X$ from a topological space $X$ to $X$, the base extension $Y \rightarrow X$ of $f$ satisfies $\mathbf{P}$.

Definition 3.1. A pre-Deligne-Mumford topological stack $X$ is called a DeligneMumford topological stack if the diagonal $X \rightarrow X \times X$ is representable by closed maps with discrete finite fibers.

Remark 3.2. In [7] the finiteness condition in the definition of a Deligne-Mumford topological stack is replaced by a weaker condition which allows infinite inertia groups.

With some extra care, the natural constructions of homotopy theory of topological spaces can be extended to Deligne-Mumford topological stacks. For instance, one can define a reasonable notion of homotopy between maps, and this allows us to define the $n$-th homotopy group of a pointed topological stack $(X, x)$ as pointed homotopy classes of maps $\left[\left(S^{n}, *\right),(\mathcal{X}, x)\right]$. 
In particular, we can talk about the fundamental group $\pi_{1}(X, x)$ of a topological stack. When $X$ is locally well-behaved (i.e. it is locally path-connected and semilocally 1-connected), the fundamental group classifies the connected covering spaces of $\mathcal{X}$ (that is, there is a natural bijection between the equivalence classes of pointed connected covering spaces of $X$ and subgroups of $\pi_{1}(X, x)$; in particular, $X$

has a universal cover $\tilde{X}$ that is unique up to equivalence, and $X$ is the quotient of $\tilde{X}$ by a natural action of $\left.\pi_{1}(X, x)\right)$. Recall from [7] that a covering space of $X$ is defined to be a representable map $y \rightarrow X$ of stacks that is $\mathbf{P}$, where $\mathbf{P}=$ covering map (see the second paragraph of this section). Note that we have not assumed $y$ to be connected. For more details on the covering theory of stacks the reader may consult [7].

To any topological stack $X$ one can associate an honest topological space $X_{\text {mod }}$, called the coarse moduli space, or more informally the underlying space, of $\mathcal{X}$. The coarse moduli space $X_{\text {mod }}$ is in some sense the best approximation of $\mathcal{X}$ by a topological space. For instance, when $X$ is a quotient stack of a group action, then $X_{\text {mod }}$ is just the usual coarse quotient. There is a natural continuous map $X \rightarrow x_{\text {mod }}$, called the moduli map. Usually we visualize a topological stack by drawing its coarse moduli space and describing the stacky structure by specifying the inertia groups of the points; of course this naive picture is not enough to recover the stack. For example, given a loop on $\mathcal{X}$, we draw it as a loop on $\mathcal{X}_{\text {mod }}$, but it should be kept in mind that different loops in $X$ may give rise to the same drawing. For example, typically we have non-trivial loops in $X$ that become constant loops in $X_{\text {mod }}$.

3.2. Complex analytic stacks. Since we are only interested in smooth analytic stacks, we give a simplistic definition of (smooth) Deligne-Mumford analytic stacks which makes life easier for those who do not feel comfortable with analytic spaces (see Remark 3.4 below).

Let Comp be the category of complex manifolds, endowed with the usual Grothendieck topology (where covers are simply topological open covers). As in the case of topological stacks, we can construct the 2-category of stacks over Comp, and, by Yoneda, this category contains the category of complex manifolds as a full subcategory.

We say a morphism $f: y \rightarrow X$ of stacks over Comp is representable by local homeomorphisms, if for any map $X \rightarrow X$ from a complex manifold $X$ to $\mathcal{X}$, the fiber product $Y=X \times x y$ is equivalent to a complex manifold, and the map $Y \rightarrow X$ is a local homeomorphism. A stack $X$ over Comp is called a (smooth) pre-Deligne-Mumford analytic stack if there exists an epimorphism $p: X \rightarrow X$ from a complex manifold $X$ such that $p$ is representable by local homeomorphisms. We say a morphism $f: y \rightarrow X$ of pre-Deligne-Mumford analytic stacks is representable, if for any map $X \rightarrow X$ from a complex manifold $X$ to $X$ that is representable by local homeomorphisms, the fiber product $Y=X \times x y$ is equivalent to a complex manifold. Let $\mathbf{P}$ be a property of morphisms of complex manifolds that is invariant under base change along local homeomorphisms. For example we can take $\mathbf{P}=$ closed, $\mathbf{P}=$ finite fibers, $\mathbf{P}=$ discrete fibers or $\mathbf{P}=$ covering space. Then, we say a representable morphism $f: y \rightarrow X$ of pre-Deligne-Mumford analytic stacks is $\mathbf{P}$, if for any map $X \rightarrow X$ from a complex manifold $X$ to $X$ that is representable by local homeomorphisms, the base extension $Y \rightarrow X$ is $\mathbf{P}$. 
Definition 3.3. A pre-Deligne-Mumford analytic stack $X$ is called a DeligneMumford analytic stack if the diagonal $X \rightarrow X \times X$ is representable by closed maps with finite fibers.

Remark 3.4. In [7] the definition of a Deligne-Mumford analytic stack is different from the one given above in two ways. Firstly, here we have added the finiteness condition on the diagonal to make the exposition simpler. Secondly, in [7] the base Grothendieck site is taken to be the category Analytic of all analytic spaces,instead of the smaller Comp. However, apart from the finiteness conditions on the diagonal, the two theories are "the same" if we restrict out attention to smooth analytic stack. In other words, the 2-category of analytic stacks defined above is equivalent to the sub 2-category of the 2-category of analytic stack of [7] consisting of smooth analytic stacks. In more technical terms, if $\mathcal{X}$ is a smooth analytic stack in the sense of [7], one can always recover $X$ from its restriction to Comp using a homotopy left Kan extension. More casually speaking, this means that, for a smooth $X$, if we know the maps from smooth domains into $X$, then we know the maps from an arbitrary domain into $X$.

We quote the following result from [7].

Proposition 3.5. Let $X$ be a Deligne-Mumford topological (resp., analytic) stack. Then there is a covering $\left\{\mathcal{U}_{i}\right\}$ of $X$ by open substacks such that each $\mathcal{U}_{i}$ is a quotient stack $[X / G]$, where $X$ is a topological space (resp., complex manifold), and $G$ a finite group acting continuously (resp., analytically) on $X$.

One can define the coarse moduli space $X_{\text {mod }}$ of an analytic Deligne-Mumford stack $\mathcal{X}$. It is an analytic space, but it may not in general be a smooth complex manifold. When $X$ is 1-dimensional, however, which is the only case we are interested in anyway, the coarse moduli space $X_{m o d}$ is an analytic curve (i.e., a Riemann surface).

3.3. Comparing algebraic, analytic and topological stacks. In any of the algebraic, analytic or topological settings, there is an alternative description of Deligne-Mumford stacks using groupoids. For instance, in the algebraic setting, a Deligne-Mumford stack $X$ can be described by (the Morita equivalence class of) an étale groupoid $X_{1} \rightrightarrows X_{0}$, where the diagonal $X_{1} \rightrightarrows X_{0} \times X_{0}$ is assumed to be a finite morphism. We have similar descriptions in the analytic and topological settings. This enables us to define natural functors

$$
\mathrm{AlgDM} \rightarrow \mathrm{AnDM} \rightarrow \text { TopDM }
$$

For instance, let $X$ be a smooth algebraic Deligne-Mumford stack of finite type over complex numbers, with $X_{1} \rightrightarrows X_{0} \times X_{0}$ an étale groupoid representing it. Then we define $X^{a n}$ to be the quotient of the groupoid $X_{1}^{a n} \rightrightarrows X_{0}^{a n} \times X_{0}^{a n}$.

We can do the same thing to get a topological stack $X^{t o p}$ from an analytic (or algebraic) stack $X$. The homotopy groups of an analytic (or algebraic) stack $\mathcal{X}$ are defined to be those of $x^{\text {top }}$.

The proof that the above functors are well-defined can be found in [7], where the reader can also find the proof of the fact that these functors respect the coarse moduli space construction. 


\section{Some useful Facts}

4.1. Basic facts about fundamental groups. In [9] it is shown that for any pointed algebraic stack $(\mathcal{X}, x)$ there is a natural group homomorphism $I_{x} \rightarrow \pi_{1}(\mathcal{X}, x)$, where the group $I_{x}$ is the inertia group of the residue gerbe at $x .{ }^{1}$ Similar statement for topological (hence also analytic) stacks is proved in [7]. We quote the following results from loc. cit.:

Lemma 4.1. Let $X$ be a Deligne-Mumford topological stack, and let $(y, y) \rightarrow(X, x)$ be a pointed covering space. Then we have a cartesian diagram

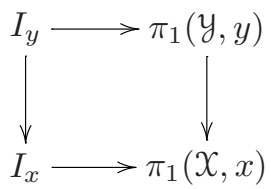

In particular, the kernel of $I_{x} \rightarrow \pi_{1}(X, x)$ is invariant under covering maps. The same statement is true for analytic and algebraic stacks (where in the algebraic situation $\pi_{1}(X, x)$ refers to algebraic fundamental group, and covering maps are understood to be finite étale maps).

Theorem 4.2. Suppose $\mathcal{X}$ is a Deligne-Mumford topological stack.

i. Let $y \rightarrow X$ be a covering space, and let $H \subseteq \pi_{1} X$ be the corresponding subgroup (for a choice of base points). Then, $y$ is an honest topological space if and only if for any point $x$, the map $I_{x} \rightarrow \pi_{1}(X, x)$ is injective and its image does not intersect any conjugate of $H$.

ii. $X$ is uniformizable (i.e. its universal cover is an honest topological space) if and only if the maps $I_{x} \rightarrow \pi_{1}(X, x)$ are injective for every point $x$ in $X$. If, furthermore, the maps $I_{x} \rightarrow \pi_{1}(X, x)$ remain injective after passing to profinite completion, then $\mathcal{X}$ is the quotient stack of a finite group acting on a topological space.

These statements remain valid for analytic and algebraic stacks (where in the algebraic situation $\pi_{1}(X, x)$ refers to the algebraic fundamental group and uniformizable means that $X$ is the quotient stack of the action of a finite group on an algebraic space).

Remark 4.3. In this paper the only algebraic spaces that will come up are smooth and 1-dimensional, in which case they are automatically schemes. So in the algebraic version of the previous theorem we will actually get smooth algebraic curves covering $x$.

The following theorem is proved in [7].

Theorem 4.4. Let $\mathcal{X}$ be an algebraic stack of finite type over $\mathbb{C}$. Then the algebraic fundamental group of $\mathcal{X}$ is isomorphic to the profinite completion of the topological fundamental group of the underlying topological stack $X^{\text {top }}$.

The van Kampen theorem remains true for topological stacks. The proof will appear elsewhere.

\footnotetext{
${ }^{1}$ In [9] the groups $I_{x}$ are named hidden fundamental groups and are denoted by $\pi_{1}^{h}(X, x)$.
} 
4.2. Stacky discs and their fundamental groups. In this part we look at (smooth) Deligne-Mumford analytic stacks whose coarse moduli space is isomorphic to $\mathbb{D}$ or $\mathbb{C}$. More specifically, we are interested in ones that are gerbes away from the origin.

The following two propositions explains why we would like to study stacky discs.

Proposition 4.5. Let $\mathfrak{U}$ be a Deligne-Mumford analytic orbifold whose coarse moduli space is $\mathbb{D}$ (resp. $\mathbb{C}$ ). Assume $\mathcal{U}$ has at most one orbifold point. Then $\mathcal{U}$ is isomorphic to $\mathcal{D}_{n}$ (resp. $\mathcal{C}_{n}$ ) for some $n \geq 1$. (See Section 2 for notation.)

Proof. We consider the case where the coarse moduli space of $\mathcal{U}$ is $\mathbb{D}$; the proof goes through similarly in the case where it is $\mathbb{C}$. We will assume that $\mathcal{U}$ is not a space (so it has a unique orbifold point).

Let $x$ be the orbifold point of $\mathcal{U}$. By Proposition 3.5, there is an open substack $\mathcal{U}^{\prime} \subset \mathcal{U}$ around $x$ that is a quotient stack by a finite group action. After shrinking $\mathcal{U}^{\prime}$, we may assume that its underlying space is a disc. Now, by Theorem 4.2, $I_{x} \rightarrow \pi_{1}\left(\mathcal{U}^{\prime}, x\right)$ is injective. But $\pi_{1}\left(\mathcal{U}^{\prime}, x\right)=\pi_{1}(\mathcal{U}, x)$. So $I_{x} \rightarrow \pi_{1}(\mathcal{U}, x)$ is injective. Hence, by Theorem $4.2, \mathcal{U}$ is uniformizable. The universal cover of $\mathcal{U}$ is necessarily either $\mathbb{D}$ or $\mathbb{C}$. But $\mathbb{C}$ is ruled out, since composing the map $\mathbb{C} \rightarrow \mathcal{U}$ with $\mathcal{U} \rightarrow$ $\mathcal{U}_{\text {mod }} \cong \mathbb{D}$ would give a surjective map $\mathbb{C} \rightarrow \mathbb{D}$ which is impossible. So we have $\mathcal{U} \cong[\mathbb{D} / G]$, where $G$ is a discrete group acting properly discontinuously on $\mathbb{D}$. Since $\mathcal{U}$ has a unique orbifold point, this action has a unique orbit $\mathcal{O}$ whose elements have non trivial stabilizers. If we remove this orbit from $\mathbb{D}$, we find a covering space of the punctured disc $\mathbb{D}^{*}$. But the only possible covering spaces of $\mathbb{D}^{*}$ are isomorphic to either $\mathbb{D}$ or $\mathbb{D}^{*}$. This implies that the orbit $\mathcal{O}$ is in fact just a single point, which after some change of coordinates can be taken to be $0 \in \mathbb{D}$. So, 0 is the unique fixed point of the action of $G$. Since $\mathcal{U}$ is a Deligne-Mumford stack, $G$ must be finite. The only finite groups of automorphisms of $\mathbb{D}$ which fix the origin are the cyclic rotation groups. So $G \cong \mathbb{Z}_{n}$, for some $n \geq 2$, and it acts by rotations around origin. Therefore, $\mathcal{U} \cong \mathcal{D}_{n}$.

This proposition implies that an analytic orbifold curve $\mathcal{C}$ is uniquely defined by giving a Riemann surface $C$ (to be $\mathrm{e}_{\text {mod }}$ ), a finite collection $A$ of points on $C$ (the orbifold points), and an integer $n_{x} \geq 2$ for each $x \in A$ (the orbifold degree at $x$ ).

Proposition 4.6. Let $\mathcal{X}$ be a Deligne-Mumford analytic stack. Then $X$ is an $H$ gerbe over an orbifold $y$, for some finite group $H$. Furthermore, the construction of $y$ is functorial in $X$, in the sense that, an arbitrary map $X^{\prime} \rightarrow X$ of Deligne-Mumford analytic stacks induces a natural map $y^{\prime} \rightarrow y$ of the corresponding orbifolds, making the following diagram commute:

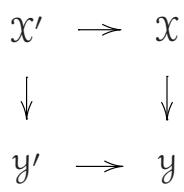

The same statement is true for algebraic and topological Deligne-Mumford stacks. (Here H-gerbe means, locally (in the étale topology) our gerbe is equivalent to the classifying stack of $H$.) 
Proof. We only give the proof for the analytic stacks because that is what we need. The exact same proof works for topological stacks, and the same idea can be made to work in the algebraic case too.

Let $R \rightrightarrows X$ be an étale groupoid representing $X$, and let $S \subseteq R$ be its stabilizer group. We claim that there is unique maximal locally constant subgroup $T$ of $S$ (as groups over $X$ ), and that $T$ is normal in $R$. Having shown this, it is enough to consider the groupoid $R^{\prime} \rightrightarrows X$, where $R^{\prime}=R / T$ is the quotient groupoid, and define $y$ to be $\left[X / R^{\prime}\right]$.

Since the statement is local we can use Proposition 3.5 and assume that $X=$ $[X / G]$, where $G$ is a finite group acting on a complex manifold. Let $H \subseteq G$ be the subgroup of elements that act trivially on $X$. It is easy to see that $H$ is normal in $G$ and that, as a subgroupoid of the stabilizer of the groupoid $G \times X \rightrightarrows X$, it is the largest locally constant subgroup. This proves the claim. The proof of functoriality is easy and is also left to the reader.

Now, suppose $\mathcal{U}$ is a Deligne-Mumford analytic stack whose coarse moduli space is $\mathbb{D}$, and that, away from the origin, is an $H$-gerbe over $\mathbb{D}^{*}$ for some finite group $H$. Let $G$ be the inertia group of $\mathcal{U}$ at the origin. By Proposition 4.6 and Proposition $4.5, \mathcal{U}$ is an $H$-gerbe over some $\mathcal{D}_{n}, n \geq 1$.

We have a short exact sequence

$$
1 \rightarrow H \rightarrow G \rightarrow \mathbb{Z}_{n} \rightarrow 1,
$$

and this short exact sequence uniquely characterizes $\mathcal{U}$ up to isomorphism (Proposition 4.7). Here is how we can reconstruct $\mathcal{U}$ from this short exact sequence: let $G$ act on $\mathbb{D}$ via its quotient $\mathbb{Z}_{n}$ (by rotations). The quotient of this action is isomorphic to $\mathcal{U}$. In particular, $\mathcal{U}$ is uniformizable, and its fundamental group is isomorphic to $G$. More generally, we have the following.

Proposition 4.7. Let $y$ be a topological stack such that $\pi_{n} y$ is trivial for $n \geq 2$. Let $H$ be a discrete group. Let $\mathcal{X}$ be an $H$-gerbe over $\mathcal{Y}$. Then, there is a short exact sequence

$$
1 \rightarrow H \rightarrow \pi_{1} x \rightarrow \pi_{1} y \rightarrow 1
$$

and this short exact sequence characterizes $X$ up to isomorphism. Furthermore, $X$ and $y$ have the same universal cover. In particular, $X$ is uniformizable if and only if $y$ is. In this case, we have $\mathcal{X} \cong\left[\tilde{y} / \pi_{1} X\right]$, where $\pi_{1} X$ acts on $\tilde{y}$ via the map $\pi_{1} x \rightarrow \pi_{1} y$ ).

Proof. The short exact sequence is simply the fiber homotopy exact sequence. To prove that it characterizes $\mathcal{X}$, it is enough to show that $\mathcal{X}$ and $y$ have the same universal cover (because then $X$ will have to be the quotient stack $\left[\tilde{y} / \pi_{1} X\right]$ ).

To prove that $x$ and $y$ have the same universal cover let us consider the following commutative diagram:

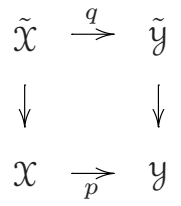

By the 2-out-of-3 property of finite étale maps, it follows that $q$ is finite étale (not, a priori, representable). Let $\mathcal{F}$ be the fiber of $q$. Since $\mathcal{F}$ is finite étale over a point, 
it can only have non-trivial $\pi_{0}$ and $\pi_{1}$. On the other hand, since all homotopies of $\tilde{y}$ vanish, the fiber homotopy exact sequence implies that the map $\mathcal{F} \rightarrow \tilde{X}$ induces an isomorphism on all $\pi_{i}, i \geq 0$. Since $\tilde{X}$ is connected and simply connected, this implies that $\pi_{i} \mathcal{F}$ is trivial for $i \geq 0$. That is, $\mathcal{F}$ is just a point. Therefore, $q$ is an isomorphism.

Observe that the short exact sequence of the proposition gives rise to an element in $\operatorname{Hom}\left(\pi_{1} y\right.$, Out $\left.H\right)$, the band of the gerbe. When $y=U$ is an open (non-orbifold) analytic curve, the second cohomology group (with coefficients in an abelian sheaf) is trivial, so an $H$-gerbe over $U$ is characterized by its band. ${ }^{2}$

Gerbes with trivial band. Now let us look more closely at gerbes over $\mathcal{D}_{n}$ whose band is trivial. The following sets are in natural bijection:

- $H$-gerbes over $\mathcal{D}_{n}$ whose band is trivial (with a chosen trivialization), up to isomorphism.

- Extensions of $\mathbb{Z}_{n}$ by $H$ for which the centralizer of $H$ surjects onto $\mathbb{Z}_{n}$, up to an isomorphism inducing identity on $H$ and $\mathbb{Z}_{n}$.

- Central extensions of $\mathbb{Z}_{n}$ by $Z(H)$, up to an isomorphism inducing identity on $Z(H)$ and $\mathbb{Z}_{n}$.

- $Z(H) / n Z(H)$.

Let us be more explicit about these correspondences because we will use them later for calculating some fundamental groups. Consider an $H$-gerbe over $\mathcal{D}_{n}$ with trivial band. The corresponding group extension

$$
1 \rightarrow H \rightarrow G \rightarrow \mathbb{Z}_{n} \rightarrow 1
$$

has the property that for any element in $\mathbb{Z}_{n}$ the corresponding outer action on $H$ is trivial (this means the band is trivial). Therefore, $G=Z_{G}(H) H$. This gives rise to the following central extension

$$
1 \rightarrow Z(H) \rightarrow Z_{G}(H) \rightarrow \mathbb{Z}_{n} \rightarrow 1 .
$$

We can recover the original extension by pushing out along $Z(H) \hookrightarrow H$. Let us now elaborate on the bijection between the extensions of $\mathbb{Z}_{n}$ by $H$ and $Z(H) / n Z(H)$. Given an element $a \in Z(H)$, define

$$
G_{a}:=H \times \mathbb{Z} /(a,-n) .
$$

We have an exact sequence

$$
1 \rightarrow H \rightarrow G_{a} \rightarrow \mathbb{Z}_{n} \rightarrow 1
$$

which is, up to an isomorphism inducing identity on $H$ an $\mathbb{Z}_{n}$, independent of the class of $a$ in $Z(H) / n Z(H)$. Conversely, given an extension

$$
1 \rightarrow H \rightarrow G \rightarrow \mathbb{Z}_{n} \rightarrow 1
$$

pick an element $g \in G$ which maps to $1 \in \mathbb{Z}_{n}$. The conjugation action of $g$ on $H$ is inner by assumption. So we can find $h \in H$ which induces the same action. Let $a:=h^{n}$. The conjugation action of $a$ on $H$ must then be trivial. That is, $a$ lies in the center of $H$. The class of $a$ in $Z(H) / n Z(H)$ is easily seen to be independent of the choice of $g$.

\footnotetext{
${ }^{2}$ Having fixed an isomorphism $\pi_{1} U \cong \mathbb{Z}^{* m}$, this amounts to specifying $m$ elements in Out $H$. We will use these elements to construct the graph of groups associated to an open Deligne-Mumford curve (see Section 7.1)
} 
Extension of a gerbe from $\mathcal{D}_{\infty}$ to $\mathcal{D}_{n}$. It is an easy exercise to check that an $H$-gerbe over $\mathcal{D}_{\infty}$ extends to an $H$-gerbe over $\mathcal{D}_{n}$ if and only if the corresponding element in $\operatorname{Out}(H)$ is $n$-torsion. The extensions of a given gerbe are acted upon simply transitively by $H^{2}\left(\mathbb{Z}_{n}, Z(H)\right)$. Here $Z(H)$ is made into a $\mathbb{Z}_{n}$-module via the action induced by the given $n$-torsion element in $\operatorname{Out}(H)$. We have the corresponding cartesian diagram of group extensions:

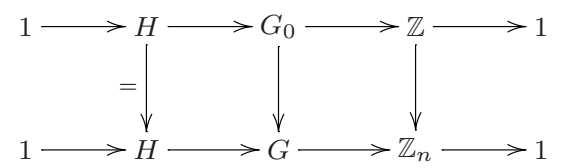

Note that $G_{0} \cong H \rtimes \mathbb{Z}$. The semidirect product is defined by choosing a lift of the given element of $\operatorname{Out}(H)$ to $\operatorname{Aut}(H)$. This isomorphism becomes canonical once we choose a lift of $1 \in \mathbb{Z}=\pi_{1} \mathcal{D}_{\infty}$ to a loop in the gerbe.

Differential forms on $\mathcal{D}_{n}$. The sheaf of differentials on $\mathcal{D}_{n}$ is a line bundle. Sections of this line bundle are the $\mathbb{Z}_{n}$-invariant differential forms on $\mathbb{D}$, necessarily of the form $z^{n-1} f\left(z^{n}\right) d z$. Therefore, $z^{n-1} d z$ is a generator of the sheaf of differentials on $\mathcal{D}_{n}$. Its order of vanishing is $\frac{n-1}{n}$.

4.3. Weighted projective lines, footballs, and drops. For any pair of integers $m, n \geq 1$ there is a Deligne-Mumford curve $\mathcal{P}(m, n)$, called the weighted projective line of type $(m, n)$. It has at most two orbifold points, and its coarse moduli space is $\mathbb{P}^{1}$. Here is the standard way of constructing $\mathcal{P}(m, n):{ }^{3}$ consider the action of $\mathbb{C}^{*}$ on $\mathbb{C}^{2}-\{0\}$ given by $t .(x, y)=\left(t^{m} x, t^{n} y\right)$. The corresponding quotient stack is $\mathcal{P}(m, n)$. In particular, we have a $\mathbb{C}^{*}$-fibration over our weighted projective line whose total space is $\mathbb{C}^{2}-\{0\}$. A fiber homotopy exact sequence argument shows that $\mathcal{P}(m, n)$ is simply connected, for all $m, n \geq 1$. In fact, $\pi_{i} \mathcal{P}(m, n) \cong \pi_{i} S^{2}$ for all $i$.

Obviously, $\mathcal{P}(m, n) \cong \mathcal{P}(n, m)$. A weighted projective line is an orbifold if an only if $m$ and $n$ are relatively prime. We call these orbifold weighted projective lines. Note that $\mathcal{P}(1,1)=\mathbb{P}^{1}$. The weighted projective line $\mathcal{P}(m, n)$ is a $\mathbb{Z}_{d^{-}}$-gerbe over the weighted projective line $\mathcal{P}\left(\frac{m}{d}, \frac{n}{d}\right)$, where $d$ is the greatest common divisor.

For a pair of integers $m, n \geq 1$, a football of type $(m, n)$ is an orbifold whose underlying space is $\mathbb{P}^{1}$ and has two orbifold points of order $m$ and $n$. We denote by $\mathcal{F}(m, n)$ the football whose underlying space is $\mathbb{P}^{1}$ and has orbifold points of order $m$ and $n$ at 0 and $\infty$, respectively. Any football of type $(m, n)$ is isomorphic to $\mathcal{F}(m, n)$. When exactly one of $m$ or $n$ is 1 , a football of type $(m, n)$ is sometimes called a drop.

When $m$ and $n$ are relatively prime, we have a natural isomorphism $\mathcal{F}(m, n) \cong$ $\mathcal{P}(m, n)$. In other cases, $\mathcal{F}(m, n)$ and $\mathcal{P}(m, n)$ are non-isomorphic.

When $m$ and $n$ are strictly greater than 1 , the group of automorphisms of $\mathcal{F}(m, n)$ is $\mathbb{C}^{*}$, the action being the obvious rotation action. When exactly one of $m$ and $n$ is equal to 1 , say $m=1$, the group of automorphisms of $\mathcal{F}(m, n)$ is naturally isomorphic with the group of affine transformations of the complex plane $\mathbb{C} \subset$ $\mathcal{F}(1, n)$.

\footnotetext{
${ }^{3}$ See Section 6 for an alternative construction
} 


\section{Uniformization of Deligne-Mumford CURVEs: The CASE OF AN ORBIFOLD}

In this section we compute the fundamental groups of orbifold analytic curves, determine the simply connected ones, and classify orbifold curves by their uniformization types. The results of this section are quite elementary, presumably well-known (see for instance [3] Section IV.9; especially, Theorem IV.9.12).

We will assume that our orbifold curves $\mathcal{C}$ are of finite type. That is, the underlying space $\mathcal{C}$ is a compact Riemann surface with a finite number of "holes". The holes can be "big" (e.g. $\mathbb{D}$ can be thought of as the Riemann sphere with a big hole), or small (e.g. $\mathbb{C}$ can be thought of as the Riemann sphere with a small hole).

We begin by stating the main results, postponing the proofs to 5.1.

Proposition 5.1. Every orbifold curve has a universal cover which is a simply connected orbifold curve. The simply connected orbifold curves are precisely $\mathbb{H}, \mathbb{C}$, and $\mathcal{P}(m, n)$ for $(m, n)=1$.

Proof. Follows from Proposition 5.7

Definition 5.2. We call an orbifold curve hyperbolic, Euclidean, or spherical if its universal cover is $\mathbb{H}, \mathbb{C}$, or $\mathcal{P}(m, n)$, respectively.

Proposition 5.3 (hyperbolic orbifolds). All non-algebraic orbifold curves are hyperbolic. An algebraic orbifold curve is hyperbolic if and only if

$$
2 g-2+\sum \frac{n_{i}-1}{n_{i}}
$$

is greater than 0 , where $n_{i} \leq \infty$ are the orders of the orbifold points of $\mathrm{C}$ (the orbifold order of a hole is $n=\infty$ ).

Every hyperbolic orbifold is the quotient of $\mathbb{H}$ by a finitely generated discrete subgroup of $\mathrm{PSL}_{2}(\mathbb{R})$. Conversely, the quotient of $\mathbb{H}$ by any such subgroup is a hyperbolic orbifold curve. Two orbifold curves are isomorphic if and only if the corresponding subgroups of $\mathrm{PSL}_{2}(\mathbb{R})$ are conjugate.

Proof. See Proposition 5.11 and the discussion afterwards.

Proposition 5.4 (Euclidean orbifolds). Besides the non-compact $\mathrm{C}_{n}$ and $\mathrm{C}_{2,2}$, and the non-orbifold genus 1 curves, the rest of the Euclidian orbifolds have coarse moduli space $\mathbb{P}^{1}$ and orbifold point orders $(3,3,3),(2,4,4),(2,3,6)$ or $(2,2,2,2)$. The fundamental groups of these are $\Lambda_{0} \rtimes \mu_{3}, \Lambda_{0} \rtimes \mu_{6}, \Lambda_{1728} \rtimes \mu_{4}$ or $\Lambda \rtimes \mu_{2}$, which act via the obvious embeddings into $\mathbb{C} \rtimes \mathbb{C}^{*}$ on $\mathbb{C}$. Here $\Lambda$ denotes a generic lattice, and $\Lambda_{0}=<1, \xi_{6}>, \Lambda_{1728}=<1, i>$ are the two symmetric lattices.

Proposition 5.5 (spherical orbifolds). These all have coarse moduli space $\mathbb{P}^{1}$. We have the following possibilities:

i. The number of orbifold points is at most 2 . In this case $\mathcal{C} \cong \mathcal{F}(m, n)$, where $n, m \geq 1$ are the orders of the orbifold points. We have $\pi_{1} \mathrm{e} \cong \mathbb{Z}_{d}$, where $d=(m, n)$, and the universal cover is $\mathcal{P}\left(\frac{n}{d}, \frac{m}{d}\right)$ on which $\mathbb{Z}_{d}$ acts by rotations.

ii. The number of orbifold points is at least 3. In this case we have the following possibilities for the orders of the orbifold points: $(2,2, n)$, for some $n \geq 2$, $(2,3,3),(2,3,4)$ or $(2,3,5)$. The corresponding fundamental group is the dihedral, tetrahedral, octahedral or icosahedral group, respectively, and the universal cover is $\mathbb{P}^{1}$, acted upon in the usual way. 
5.1. Proofs. We begin with computing the fundamental group of an arbitrary orbifold curve. Let $\mathcal{C}$ be an orbifold curve. Let $P_{1}, \cdots, P_{k}$ be its orbifold points, with $n_{1}, \cdots, n_{k}<\infty$ their orders, and let $Q_{1}, \cdots, Q_{l}$ be the holes. Assume the genus of the underlying (compactified) Riemann surface is $g$, and $l+k \geq 1$.

If we remove the $P_{i}$ we obtain an open Riemann surface $U$ whose fundamental group is isomorphic to $\mathbb{Z}^{*(2 g+k+l-1)}$. More precisely, let $a_{1}, b_{1}, \cdots, a_{g}, b_{g}$ be the generators of $\pi_{1} \mathrm{C}_{\text {mod }}, \rho_{j}$ loops around $P_{j}$, and $\sigma_{h}$ loops around $Q_{h}$. Then,

$$
\pi_{1} U \cong\left(* \mathbb{Z} a_{i}\right) *\left(* \mathbb{Z} b_{i}\right) *\left(* \mathbb{Z} \rho_{j}\right) *\left(* \mathbb{Z} \sigma_{h}\right) /<\prod\left[a_{i}, b_{i}\right] \prod \rho_{j} \prod \sigma_{h}=1>.
$$

If we stick the $P_{j}$ back on, we introduce the new relations $\left(\rho_{j}\right)^{n_{j}}=1$, for $1 \leq j \leq k$. From van Kampen we obtain the following

Proposition 5.6. There is an isomorphism

$$
\pi_{1} \mathcal{C} \cong\left(* \mathbb{Z} a_{i}\right) *\left(* \mathbb{Z} b_{i}\right) *\left(* \mathbb{Z}_{n_{j}} \rho_{j}\right) *\left(* \mathbb{Z} \sigma_{i}\right) /<\prod\left[a_{i}, b_{i}\right] \prod \rho_{j} \prod \sigma_{h}=1>.
$$

Proposition 5.7. Let $\mathcal{C}$ be an orbifold curve. If $\mathcal{C}$ is a football $\mathcal{F}(m, n)$ with $m \neq n$, then the universal cover of $\mathcal{C}$ is $\mathcal{F}\left(\frac{m}{d}, \frac{n}{d}\right)$, where $d=\operatorname{gcd}(m, n)$. In particular, $\mathcal{C}$ is not uniformizable. In the remaining cases, $\mathrm{C}$ is uniformizable. More strongly, we have $\mathcal{C} \cong[X / G]$, where $X$ is a Riemann surface, and $G$ is finite group acting on it.

Proof. Let the notation be as in the paragraph preceding the proposition. We consider several cases (A-E below). In cases A-D we show that the injectivity condition of Theorem 4.2 is satisfied, so $\mathcal{C}$ is uniformizable. In fact, we show more strongly that this injectivity condition is satisfied for the profinite completion of the $\pi_{1} \mathrm{C}$. So, in cases A-D, $\mathcal{C}$ is in fact the quotient stack of a finite group acting on a Riemann surface $C$. Note that, if $\mathcal{C}$ is algebraic in any of these cases, then $C$ will be algebraic too.

Case $\mathrm{E}$ is the only case in which $\mathcal{C}$ is not uniformizable. In this case the universal cover is shown to be $\mathcal{P}(m, n)$ for some relatively prime natural numbers $m, n$.

Case $A: g \geq 1$. By passing to a quotient, we can reduce to the case $k=1, l=0$. We show that there is a finite quotient of the group

$$
A=\mathbb{Z} a * \mathbb{Z} b * \mathbb{Z}_{n} c /<a b a^{-1} b^{-1}=c>.
$$

into which $\mathbb{Z}_{n} c$ injects. We construct this group as follows. Consider the action of $\mathbb{Z}_{n} x$ on $\mathbb{Z}_{n} z \oplus \mathbb{Z}_{n} y$ where the action of the generator $x \in \mathbb{Z}_{n} x$ is given by the matrix

$$
\left(\begin{array}{ll}
1 & 1 \\
0 & 1
\end{array}\right)
$$

The semi-direct product $\left(\mathbb{Z}_{n} z \oplus \mathbb{Z}_{n} y\right) \rtimes \mathbb{Z}_{n} x$, the Heisenberg group over $\mathbb{Z}_{n}$, is made into a quotient of $A$ by sending $a, b$ and $c$ to $x, y$ and $z$, respectively. It is easy to check that this quotient has the desired property.

Case B: $g=0, l \geq 1$. If $k=0$ the statement is trivial. The case $k \geq 1$ is reduced to the case $k=l=1$ by passing to a quotient, in which case the statement is again obvious.

Case $C: g=0, l=0$ and $k \geq 3$. Again, by passing to a quotient, we may assume $k=3$. For any triple of integers $m, n, p \geq 2$, Fox [2] constructs two permutations $a, b$ in a certain finite permutation group such that $a$ has order $m, b$ has order $n$, and $a b$ has order $p$. This can be used to construct the desired quotient (also see Remark 5.8). 
Case D: $g=0, l=0$, and $k=2$; equal orbifold orders. Let $n$ be the orbifold order of the two orbifold points. Then $\mathcal{C}$ is isomorphic to the quotient of $\mathbb{P}^{1}$ under the rotation action of $\mathbb{Z}_{n}$.

Case $E: g=0, l=0$, and $k=1,2$; unequal orbifold orders. When $k=1$, the resulting orbifold, a drop, is simply connected (more generally, every $\mathcal{P}(m, n)$ is simply connected). The last case is $k=2$. Assume the orbifold points have order $m \neq n$. Then, the fundamental group is isomorphic to $\mathbb{Z}_{d}$, where $d$ is the greatest common divisor of $m$ and $n$. This easily follows from van Kampen. The universal cover of $\mathcal{F}(m, n)$ is $\mathcal{P}\left(\frac{m}{d}, \frac{n}{d}\right)$; the action of $\mathbb{Z}_{d}$ on $\mathcal{P}\left(\frac{m}{d}, \frac{n}{d}\right)$ is by $\frac{2 k \pi}{d}$ rotations that fix the two orbifold points.

Remark 5.8. There is an alternative method to prove the last case $(g=0, l=0$, and $k=1,2)$ of the above proposition. It can be shown that, for any triple integers $(m, n, p)$ greater than or equal to 2 , and for any algebraically closed field $K$ whose characteristic does not divide these integers, there are elements $x, y$ in $\operatorname{PSL}(2, K)$ such that $x$ has order $m, y$ has order $n$, and $x y$ has order $p$. The group generated by $x$ and $y$ will be the desired finite quotient of our group.

To prove the existence of $x$ and $y$, we think of them as automorphisms of $\mathbb{P}_{K}^{1}$. We take $x$ to be the multiplication by a primitive $m$-the root of unity (a rotation fixing 0 and $\infty$ ), and $y$ to be a rotation of order $n$ fixing 1 and a variable point $v \in \mathbb{P}_{K}^{2}$. The condition that the matrix corresponding to $x y$ has order $p$ will be a single non-trivial equation in $v$ which can be stated by requiring that the ratio of the eigenvalues of the matrix $x y$ is a primitive $p$-root of unity. This can always be solved.

Proposition 5.7 immediately implies Proposition 5.1. Proposition 5.1 can also be proved directly. We outline the proof. Assume $\mathcal{C}$ is simply connected. Note that the coarse moduli space $\mathcal{C}_{\text {mod }}$ of $\mathcal{C}$ must be simply connected too (otherwise a non-trivial covering space of $\mathcal{C}_{\text {mod }}$ pulls back to a non-trivial covering space of $\mathcal{C}$ which contradict simple connectivity of $\mathcal{C}$ ); so $\mathcal{C}_{\text {mod }}$ is isomorphic to $\mathbb{C}, \mathbb{H}$, or $\mathbb{P}^{1}$. Therefore, $\mathcal{C}$ is one of these spaces, with a bunch of orbifold points on it. The fundamental group of such an orbifold is easily computed using van Kampen, and one checks that the only possibilities for $\mathcal{C}$ are $\mathbb{C}, \mathbb{H}$ and orbifold weighted projective lines $\mathcal{P}(m, n)$, for $m$ and $n$ relatively prime.

The following corollaries also follow easily from Proposition 5.7.

Corollary 5.9. An orbifold curve is a finite group quotient (of a Riemann surface) if and only if its universal cover is $\mathbb{H}, \mathbb{C}$ or $\mathbb{P}^{1}$ (i.e., if and only if it is a discrete group quotient).

The following is the (orientable case of the) famous Fenchel conjecture which was proved by Fox in 1952 (see pages 143-144 of [10]).

Corollary 5.10. Let $G$ be a group that is isomorphic to the fundamental group of an orbifold (e.g., any group of the form discussed in Proposition 5.6). Then, G has a subgroup of finite index that is torsion free.

Proof. If $g \geq 1$, or $l \geq 1$, or $k \geq 3$, then $G$ is isomorphic to the fundamental group of a uniformizable Deligne-Mumford curve. So by Corollary $5.9 \mathrm{G}$ has a torsion free subgroup of finite index. (Note that the fundamental group of a Riemann surface is torsion free as it acts without fixed points on a 1-dimensional complex domain.) 
If $g=l=0$ and $k \leq 2, G$ is the fundamental group of a football, which is a finite cyclic group.

Proposition 5.11. Let $\mathcal{C}$ be an orbifold curve with underlying analytic curve $C$.

i. If $C$ is compact of genus $g$, then $\mathcal{C}$ is hyperbolic (Euclidean, spherical) if and only if

$$
2 g-2+\sum \frac{n_{i}-1}{n_{i}}
$$

is greater than 0 (equal to 0 , less than 0 ). Here $n_{i}<\infty$ are the orders of the orbifold points of $\mathrm{C}$.

ii. If $C$ is not compact, then $\mathcal{C}$ is hyperbolic, unless $\mathcal{C}=\mathcal{C}_{n}, 1 \leq n \leq \infty$, or $\mathrm{e}=\mathcal{C}_{2,2}$, in which cases it is Euclidean.

Proof. If $\mathcal{C}$ is a football $\mathcal{F}(m, n)$, the assertion is obvious. So we may assume $\mathcal{C}$ is uniformizable (Proposition 5.7).

First we show that the only non-compact orbifolds whose universal cover is $\mathbb{C}$ are $\mathcal{C}_{n}, 1 \leq n \leq \infty$, and $\mathcal{C}_{2,2}$. We can argue as follows. First of all, the trivial action gives $\mathfrak{C}_{1}=\mathbb{C}$. Let $G$ be a non-trivial discrete group of automorphism of $\mathbb{C}$. Then $G$ consists of translations and rotations. If we only have translation, the possibilities (up to isomorphism) are $G \cong \mathbb{Z}$ and $G \cong \mathbb{Z} \oplus \mathbb{Z}$. The latter case is excluded since it gives a compact quotient. The former gives $\mathcal{C}_{\infty}$. If we only have rotations, then the only possibilities are finite cyclic groups of rotations around a fixed point. These give $\mathcal{C}_{n}, 2 \leq n<\infty$. Now assume $G$ contains both translations and rotations. The group of all translations in $G$ is isomorphic to $\mathbb{Z}$, and without loss of generality we can assume it is $\mathbb{Z}$ itself (i.e. translation by integers). Conjugation by any rotation in $G$ takes must take $\mathbb{Z}$ to itself. From this it easily follows that a rotation in $G$ has order two and it is centered at a point in $\frac{1}{2} \mathbb{Z}$. There are two orbifold orbits under this action, namely, $\mathbb{Z}$ and $\frac{1}{2}+\mathbb{Z}$. The quotient is $\mathcal{C}_{2,2}$.

The rest of non-compact orbifolds are uniformized by $\mathbb{H}$.

Now, assume $\mathcal{C}$ is a compact uniformizable orbifold. Choose a holomorphic differential form $\omega$ on $\mathcal{C}$. We compute the degree of $\omega$ as follows: if there were no orbifold points, then of course $\operatorname{deg}(\omega)=2 g-2$. But an orbifold pint of order $n$ has a contribution of $\frac{n-1}{n}$ (see the end Section of 4.2). Therefore,

$$
\operatorname{deg}(\omega)=2 g-2+\sum \frac{n_{i}-1}{n_{i}} .
$$

Using the fact that $\mathcal{C}$ is a finite group quotient (Corollary 5.9), it is easily seen that if this degree is positive (resp., zero, negative), then the universal cover is $\mathbb{H}$ (resp., $\left.\mathbb{P}^{1}, \mathbb{C}\right)$.

It is easy to determine when the expression $2 g-2+\sum \frac{n_{i}-1}{n_{i}}$ is negative, zero, or positive. Having done that, Propositions 5.3, 5.4, and 5.5 follow immediately from Proposition 5.11. In Proposition 5.3, the fact that the quotient of $\mathbb{H}$ under the action of any finitely generated discrete subgroup is on orbifold of finite type is not quite trivial. It follows from Ahlfors Finiteness Theorem (see [6]).

5.2. Digression on group presentations. We have shown that the fundamental group of a compact orbifold whose underlying space is a Riemann surface of genus $g$ has a presentation of the following form: 


$$
G=\left\langle a_{1}, b_{1}, \cdots a_{g}, b_{g}, c_{1}, \cdots, c_{k} \quad \mid \begin{array}{l}
c_{1}^{n_{1}}=c_{2}^{n_{2}}=\cdots=c_{k}^{n_{k}}=1 \\
{\left[a_{1}, b_{1}\right]\left[a_{2}, b_{2}\right] \cdots\left[a_{g}, b_{g}\right] c_{1} c_{2} \cdots c_{k}=1}
\end{array}\right\rangle
$$

Conversely, given such a presentation we can construct an orbifold (by attaching copies of $\mathcal{D}_{n}$ s to a bouquet of $2 g+k$ circles) whose fundamental group is isomorphic to $G$.

Groups defined by such presentations are called $F$-groups and have been the subject of intense study since the beginning of the twentieth century. It is unlikely that our methods give any new results (as perhaps there is nothing left to prove anyway!) but they do give very clean, short, and conceptual proofs of certain well known non-trivial facts about $F$-groups (see for instance Corollary 5.10). We will not pursue this further here and confine ourselves to a few words on triangle groups. ${ }^{4}$

From the uniformization theorem, we know for what values of $g, k, n_{1}, \cdots, n_{k}$ such groups $G$ are finite. For instance, when $g=0$ and $k=3$, we recover the finiteness theorems about triangle groups: it is well-known that for the values

$$
(2,2, n),(2,3,3),(2,3,4),(2,3,5)
$$

these groups are finite (the so-called, spherical triangle groups). These corresponds to orbifolds whose universal cover is $\mathbb{P}^{1}$. For

$$
(3,3,3),(2,4,4),(2,3,6)
$$

we obtain the Euclidean triangle groups which correspond to the orbifolds whose universal cover is $\mathbb{C}$ (these are of course infinite). For other values of $\left(n_{1}, n_{2}, n_{3}\right)$ we get the hyperbolic triangle groups. These ones embed as discrete subgroups of $\operatorname{PSL}(2, \mathbb{R})$. It is well-known that any hyperbolic triangle group (or in fact, any infinite $F$-group) has a subgroup of finite index that is torsion free. This corresponds to the fact that the corresponding orbifold has a finite étale cover that is a Riemann surface.

In general, any finitely generated discrete subgroup $G$ of $\operatorname{PSL}(2, \mathbb{R})$ is the fundamental group of an orbifold (Ahlfors Finiteness Theorem), hence it has a presentation of the above form. If the fundamental domain is a bounded polygon, the corresponding orbifold will be compact and algebraic. If we allow cusps as vertices of the polygon, the corresponding orbifold will still be algebraic but non-compact. In other cases our orbifold will only be analytic. In the latter two cases $G$ will indeed be isomorphic to a finite free product of cyclic groups (because the fundamental group of a non-compact orbifold curve is so). Conversely, every finite free product of cyclic groups occurs as the fundamental group of an orbifold (hence, embeds as a discrete subgroup of $\operatorname{PSL}(2, \mathbb{R}))$.

\section{Interlude: Gerbes over orbifold Weighted ProjeCtive Lines}

To prepare for the uniformization of general Deligne-Mumford curves (Section 7 ), in this section we study gerbes over weighted projective lines. We show that the only simply connected Deligne-Mumford curves whose coarse moduli space is $\mathbb{P}^{1}$ and have at most two orbifold points are weighted projective lines (Corollary $6.3)$.

\footnotetext{
${ }^{4}$ Some authors call these von Dyke groups.
} 
Let $\mathcal{P}(m, n)$ be an orbifold weighted projective line $(\operatorname{sog} \operatorname{gcd}(m, n)=1)$. Let $H$ be a finite group, and let $A=Z(H)$ be its center. Our plan is to study DeligneMumford curves $\mathcal{X}$ that are $H$-gerbes over $\mathcal{P}(m, n)$, and compute their fundamental groups. What we will do is to make explicit Giraud's classification of gerbes over $\mathcal{P}(m, n)$ in terms of their band, an element in $H^{1}(\mathcal{P}(m, n)$, Out $(H))$, and a cohomology class in $H^{2}(\mathcal{P}(m, n), A)$. Notice that, since $\mathcal{P}(m, n)$ is simply connected, any $H$-gerbe over it has a trivial band. Therefore, the set $\Gamma$ of isomorphism classes of gerbes with a trivialized band is in natural bijection with $H^{2}(\mathcal{P}(m, n), A) \cong A$. Our explicit construction of this bijection enables us to compute the fundamental groups of these gerbes. Throughout this section $m, n$ and $H$ are fixed.

Let us describe how the map $\alpha: \Gamma \rightarrow A$ is defined. Let $U, V \subset \mathcal{P}(m, n)$ be the complements of the orbifold points of order $m$ and $n$, respectively. Fix a base point * on $W=U \cap V$, and an isomorphism $\pi_{1} W \rightarrow \mathbb{Z}$. Let $X$ be an $H$-gerbe over $\mathcal{P}(m, n)$ with a trivialized band, and let $\mathcal{U}, \mathcal{V}$ and $\mathcal{W}$ denote its restrictions to $U, V$ and $W$, respectively. Observe that, since $\mathcal{W}$ has trivial band, it is indeed a trivial gerbe over $W$. Fix a lift of $*$ to $X$ (the bijection $\Gamma \rightarrow A$ will be independent of the choice of $*$ and its lift to $X)$. Pick an element $z$ in the center of $\pi_{1} \mathcal{W}$ which maps to $1 \in \pi_{1} W=\mathbb{Z}$. Let $x$ be the image of the $n^{t h}$ power of $z$ in $G=\pi_{1} \mathcal{U}$, and let $y$ be the image of the $m^{\text {th }}$ power of $z^{-1}$ in $G^{\prime}=\pi_{1} \nu$. These elements both lie in the center of $H$, namely in $A$. Since everything takes place inside the abelian group $A$ we will use the additive notation. We define $\alpha(\mathcal{X}):=m x+n y \in A$. If instead of $z$ we pick another element $z^{\prime}$ (necessarily of the form $z+a$, for some $a \in A \subseteq H \subseteq \pi_{1} \mathcal{W}$ ), we would get the pair $x+n a$ and $y-m a$, which gives rise to the same element $m(x+n a)+n(y-m a)=m x+n y$. So the map $\alpha: \Gamma \rightarrow A$ is well-defined.

Next, we construct the inverse $\gamma: A \rightarrow \Gamma$. Fix a pair of integers $r$ and $s$ such that $r n+s m=1$. Let $a$ be an element in $A$. Let $\mathcal{U}$ be the gerbe over $U$ associated to the class of $s a$ in $A / n A$ (see Section 4). Similarly, let $\mathcal{V}$ be the gerbe over $V$ associated to the class of $r a$ in $A / m A$. We want to glue these gerbes together to get a gerbe over $\mathcal{P}(m, n)$. We have the following commutative diagrams:
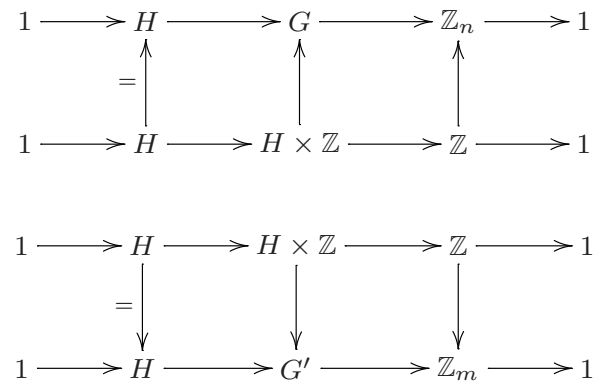

Here $G:=H \times \mathbb{Z} /(s a,-n)=\pi_{1} \mathcal{U}$. Similarly, $G^{\prime}:=H \times \mathbb{Z} /(r a,-m)=\pi_{1} \mathcal{V}$. The $H \times \mathbb{Z}$ that appears in the bottom row of the first diagram is identified with $\pi_{1}\left(\left.\mathcal{U}\right|_{W}\right)$, and the one on the top row of the second diagram is identified with $\pi_{1}\left(\left.\mathcal{V}\right|_{W}\right)$. Gluing $\mathcal{U}$ and $\mathcal{V}$ along $W$ (in a way that the trivialization of the bands is preserved) is the same as giving an isomorphism from the bottom sequence of the first diagram to the top sequence of the second diagram (inducing identity on the two ends). We do this via the map that is identity on $H$ and multiplication by -1 on $\mathbb{Z}$. This produces the desired $H$-gerbe $\gamma(a)$.

It is straightforward to check that $\alpha$ and $\gamma$ are inverse to each other. 
It would be illuminating to compare the above construction with the MayerVietoris exact sequence relative to the open cover $\{U, V\}$ (cohomologies with coefficients in $A)$ :

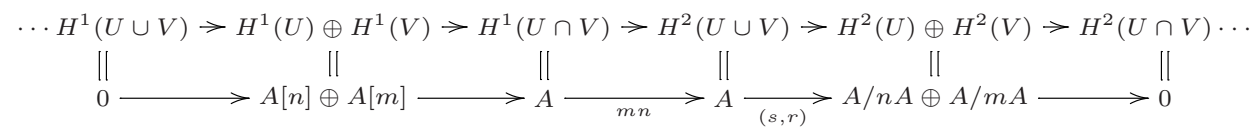

Now, let $a$ be an element in $A$ and $X$ its corresponding gerbe. Our next claim is that there is a natural isomorphism $\pi_{1} X \cong H / a$. For this we use the explicit construction of the gerbe $\gamma(a)$ as described above. We will apply van Kampen to the covering $\mathcal{U}, \mathcal{V}$ of $\mathcal{X}$. The van Kampen diagram looks as follows:

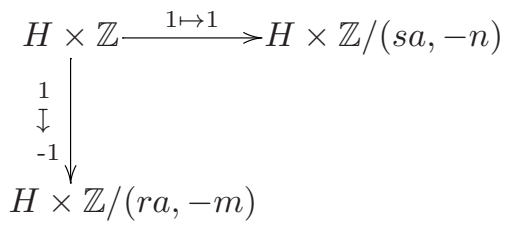

It is easy to check that the corresponding pushout is naturally isomorphic to $H / a$.

Summarizing, we have the following

Proposition 6.1. Let $m, n$ be relatively prime positive integers. Let $H$ be a finite group, and $A$ its center. Then, the isomorphism classes of $H$-gerbes $\mathcal{X}$ over $\mathcal{P}(m, n)$ with a trivialized band are in natural bijection with $A$. Furthermore, we have a natural isomorphism $\pi_{1} X \cong H / a$, where $a \in A$ is the element corresponding to $X$.

The element $a$ has an interpretation in terms of the homotopy fiber exact sequence of the fibration $B H \rightarrow \mathcal{X} \rightarrow \mathcal{P}(m, n)$ :

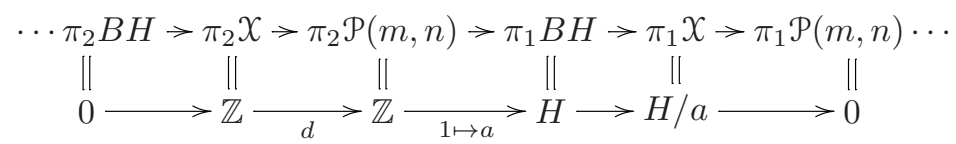

Here, $d$ is the order of $a$ in $H$. We also see from the above exact sequence that $\pi_{2} X$ is naturally isomorphic to $\mathbb{Z}$ and maps to $d \mathbb{Z} \subseteq \mathbb{Z}=\pi_{2} \mathcal{P}(m, n)$. The higher homotopy groups $\pi_{i}(\mathcal{X}), i \geq 3$, map isomorphically to $\pi_{i} \mathcal{P}(m, n)$, which are themselves isomorphic to $\pi_{i} S^{2}$. (To prove the last statement, use the homotopy fiber exacts sequence for $\left.\mathbb{C}^{2}-\{0\} \rightarrow \mathcal{P}(m, n)\right)$.

So far all the gerbes under consideration came with a fixed trivialization of their band. However, we are only interested in classification of the underlying stack of these gerbes. To this end, we have to mod out by the $\operatorname{action}$ of $\operatorname{Out}(H)$ on $A$.

Proposition 6.2. Let $m, n$ be relatively prime positive integers. Let $H$ be a finite group. Then there is a natural bijection

$\{\text { Algebraic stacks } \mathcal{X} \text { that are } H \text {-gerbes over } \mathcal{P}(m, n)\}_{\text {iso }} \rightleftharpoons \operatorname{Out}(H) \backslash A$.

The fundamental group of the stack $X_{a}$ corresponding to an element $a \in A$ is (noncanonically) isomorphic to $\mathrm{H} / \mathrm{a}$.

By the above proposition, if we want our gerbe to be simply connected, we have to assume $H$ is cyclic and $a$ is a generator for it. On the other hand, the set of generators of a cyclic group $H$ is acted on transitively by $\operatorname{Out}(H)$. Therefore, for 
a fixed cyclic group $H \cong \mathbb{Z}_{d}$ there is a unique Deligne-Mumford gerbe that is an $H$-gerbe over the weighted projective line $\mathcal{P}(m, n)$. It is nothing but the weighted projective line $\mathcal{P}(m d, n d)$ (see Section 4 ).

Corollary 6.3. Let $X$ be a Deligne-Mumford curve whose coarse moduli space is $\mathbb{P}^{1}$ and has at most two orbifold points. Then, $X$ is simply connected if and only if is isomorphic to a weighted projective line $\mathcal{P}(m, n)$.

\section{Uniformization of Deligne-Mumford curves: the general case}

In this section we extend the classification of Deligne-Mumford orbifolds by their uniformization type (Section 5) to all Deligne-Mumford curves. We saw in the previous section that in this case we have a bigger collection of simply connected Deligne-Mumford curves. Namely, we have to bring into play all $\mathcal{P}(m, n)$ for $m, n \geq$ 1. In fact, it turns out that there is no more to add (Proposition 7.2).

Let $X$ be an arbitrary Deligne-Mumford curve. Then $X$ is an $H$-gerbe over an orbifold $\mathcal{C}$. The orbifold $\mathcal{C}$ is easy to describe: it has the same coarse moduli space as $X$, and at any orbifold point of $X$ where the inertia degree jumps, say by $n$, it has an orbifold point of order $n$.

To classify simply connected Deligne-Mumford curves, we need the following simple lemma.

Lemma 7.1. Let $X$ and $y$ be connected topological stacks, and assume $X$ is an $H$-gerbe over $y$ for some discrete group $H$. Assume $X$ is simply connected. Then so is $\mathrm{y}$.

Proof. This follows immediately from the homotopy fiber sequence for the fibration $x \rightarrow y$. Alternatively, let $\tilde{y}$ be the universal cover of $y$ and set $x^{\prime}:=\tilde{y} \times y$. Then $X^{\prime} \rightarrow X$ is a covering map between connected stacks, with $X$ simply connected, so it is an isomorphism. This implies that $\tilde{y} \rightarrow y$ is also an isomorphism.

Proposition 7.2. Every Deligne-Mumford curve has a universal cover which is a simply connected Deligne-Mumford curve. The simply connected Deligne-Mumford curves are precisely $\mathbb{H}, \mathbb{C}$ and $\mathcal{P}(m, n)$, for arbitrary $n, m \geq 1$.

Proof. Assume $\mathcal{X}$ is an $H$-gerbe over an orbifold $\mathcal{C}$. By Proposition 7.1, $\mathcal{C}$ is simply connected. If $\mathcal{C}$ is $\mathbb{C}$ or $\mathbb{H}$, then $X \cong \mathcal{C}$, as there is no non-trivial gerbe over a contractible space. Otherwise, $\mathcal{C}$ is a football, in which case the result follows from Corollary 6.3.

Definition 7.3. We call a Deligne-Mumford curve hyperbolic, Euclidean or spherical, if its universal cover is $\mathbb{H}, \mathbb{C}$ or a $\mathcal{P}(m, n)$, respectively.

Proposition 7.4. Let $\mathcal{X}$ be a Deligne-Mumford curve with underlying orbifold curve $\mathcal{C}$. Then $\mathcal{X}$ is hyperbolic (Euclidean, spherical) if and only if $\mathcal{C}$ is so.

Proof. The case where $\mathcal{C}$ is hyperbolic or Euclidean follows from Proposition 4.7, as in this case $\pi_{n} \mathcal{C}$ vanishes for $n \geq 2$. When $\mathcal{C}$ is spherical, the composite map $\tilde{x} \rightarrow x \rightarrow \mathcal{C}$ induces a surjective map

$$
\tilde{x}_{\text {mod }} \rightarrow \mathcal{C}_{\text {mod }} \cong \mathbb{P}^{1}
$$

on the coarse moduli spaces. So, $\tilde{x}$ can not be any of $\mathbb{C}$ or $\mathbb{H}$. Therefore, $X$ is spherical. 
Proposition 7.5 (hyperbolic and Euclidean curves). Assume $X$ is a hyperbolic or Euclidean Deligne-Mumford analytic curve. Let $\mathcal{C}$ be the orbifold over which $\mathcal{X}$ is a gerbe, and let $H$ be the generic inertia group of $\mathcal{X}$. We have the short exact sequence

$$
1 \rightarrow H \rightarrow \pi_{1} X \rightarrow \pi_{1} \mathrm{e} \rightarrow 1
$$

Furthermore, the orbifold $\mathcal{C}$ together with the above exact sequence uniquely determine $\mathcal{X}$ up to isomorphism. More precisely, we have $\mathcal{X} \cong\left[\tilde{\mathcal{C}} / \pi_{1} X\right]$, where $\pi_{1} X$ acts on $\tilde{\mathcal{C}}$ via $\pi_{1} \mathrm{C}$.

Proof. Follows from Proposition 4.7.

Proposition 7.6 (spherical curves). Assume $X$ is a spherical Deligne-Mumford analytic curve. Let $\mathcal{C}$ be the orbifold over which $\mathcal{X}$ is a gerbe, and let $H$ be the generic inertia group of $X$.

i. $\operatorname{ker}\left(H \rightarrow \pi_{1} X\right)$ is cyclic, say of order $d$, and we have an exact sequence

$$
1 \rightarrow \mathbb{Z}_{d} \rightarrow H \rightarrow \pi_{1} \mathrm{X} \rightarrow \pi_{1} \mathrm{e} \rightarrow 1 .
$$

ii. If $\mathcal{C}$ has orbifold type $(m, n)$, then the universal cover of $\mathcal{X}$ is isomorphic to $\mathcal{P}\left(d \frac{m}{a}, k \frac{n}{a}\right)$, where $a=\operatorname{gcd}(m, n)$.

iii. If $\mathcal{C}$ has orbifold type $(2,2, n),(2,3,3),(2,3,4)$ or $(2,3,5)$, then the universal cover of $\mathcal{X}$ is isomorphic to $\mathcal{P}(d, d)$.

Proof. The exact sequence is simply the fiber homotopy exact sequence of the fibration $X \rightarrow \mathcal{C}$ (note that $\pi_{2} \mathcal{C} \cong \mathbb{Z}$ ). This proves Part (i).

Part (ii). Let $K$ be the kernel of $H \rightarrow \pi_{1} X$ (so $K \cong \mathbb{Z}_{d}$ ), and let $d=\operatorname{gcd}(m, n$ ). Let $\tilde{X}$ be the universal cover of $\mathcal{X}$. We have to show that $\tilde{X} \cong \mathcal{P}\left(d \frac{m}{a}, d \frac{n}{a}\right)$. We know that $\tilde{X}$ is an $H^{\prime}$-gerbe over a simply connected orbifold $\tilde{\mathcal{C}}$ which maps via a covering map to $\mathcal{C}$ (hence the notation $\tilde{\mathcal{C}}$ !). So we have $\tilde{\mathcal{C}} \cong \mathcal{P}\left(\frac{m}{a}, \frac{n}{a}\right)$. On the other hand, we know by Lemma 4.1 that the kernel of $H^{\prime} \rightarrow \pi_{1} \tilde{X}=1$ is the same as the kernel of $H \rightarrow \pi_{1} X$; hence, $H^{\prime} \cong \mathbb{Z}_{d}$. Therefore, $\tilde{X} \cong \mathcal{P}\left(d \frac{m}{a}, d \frac{n}{a}\right)$, by Corollary 6.3 .

Part (iii). Using an argument similar to the previous part, it follows that the universal cover of $X$ is the (unique) simply connected $\mathbb{Z}_{d}$-gerbe over $\mathbb{P}^{1}$ This is isomorphic to $\mathcal{P}(d, d)$.

Corollary 7.7. Let $X$ be a uniformizable Deligne-Mumford curve. Then there exists a Riemann surface $X$ and a finite group $G$ such that $X \cong[X / G]$.

Proof. When $X$ is spherical the result is obvious. So we assume $X$ is not spherical. It is enough to show that there exists a Riemann surface $X$ with a finite covering map $X \rightarrow X$. Assume $X$ is an $H$-gerbe over an orbifold $\mathcal{C}$. It is enough to show that there exists a finite covering map $X \rightarrow \mathcal{e}$, with $X$ Riemann surface, such that the induced $H$ gerbe on $X$ is trivial.

By Proposition 7.4, $\mathrm{C}$ is uniformizable. So, by Corollary 5.9, there is a finite covering map $C \rightarrow \mathcal{C}$. So, by pulling back our $H$-gerbe to $C$, we are reduced to the case wehere $\mathcal{C}=C$ is a Riemann surface. Recall now that an $H$-gerbe over $C$ is characterized by its band, which is an element $\beta \in H^{1}(C$, Out $(H)) \cong$ $\operatorname{Hom}\left(\pi_{1} C\right.$, Out $\left.(H)\right)$, and an element in $\alpha \in H^{2}(C, Z(H))$. Observe that $\beta$ is the homomorphism $\beta: \pi_{1} C \rightarrow \operatorname{Out}(H)$ induced from the exact sequence of Proposition 7.5. We can easily kill $\beta$ by passing to a finite cover of $C$. (For instance, let $K$ be 
the kernel of the homomorphism $\pi_{1} C \rightarrow \operatorname{Out}(H)$, and replace $C$ by the covering space $X \rightarrow C$ corresponding to the subgroup $K \subseteq \pi_{1} C$.)

To show that we can kill $\alpha$, we show that, in general, for any finite abelian group $A$, any element $\alpha \in H^{2}(C, A)$ can be killed after passing to a finite cover $f: X \rightarrow C$. If $C$ is not compact, $H^{2}(C, A)$ is already trivial and there is nothing prove. So assume $C$ is compact. It is an easy exercise that $H^{2}(C, A) \cong A$ and the map $f^{*}: H^{2}(C, A) \rightarrow H^{2}(X, A)$ is simply multiplication by $d$, where $d$ is the degree of $f$. Since $\alpha \in H^{2}(C, A)$ is torsion, say $n \alpha=0$, it is enough find such an $f$ of degree $n$. Equivalently, we have to show that the fundamental group $\pi_{1} C \cong\left(* \mathbb{Z} a_{i}\right) *\left(* \mathbb{Z} b_{i}\right) /<\prod\left[a_{i}, b_{i}\right]=1>$ has a subgroup of index $n$. Note that genus of $C$ is at least 1 (because $\mathcal{X}$, and so $\mathcal{C}$ as well, are not spherical). It is easy to see that the subgroup generated by $\left\{a_{1}, \cdots, a_{g}, b_{1}^{n}, b_{2}, \cdots, b_{g}\right\}$ is such a subgroup. This completes the proof.

As we see in Proposition 7.6, spherical Deligne-Mumford curves are more difficult to classify than their Euclidean and hyperbolic counterparts: in the hyperbolic and Euclidean cases, the exact sequence of Proposition 7.5 completely classifies the curve in question; but in the spherical case the exact sequence of Proposition 7.6 is not enough to classify the corresponding Deligne-Mumford curve. For instance, part of the information that is missing in the exact sequence of Proposition 7.6 is that the homomorphism $H \rightarrow \pi_{1} X$ carries a natural crossed-module structure; this is a general fact about fundamental groups of gerbes whose proof will not be given here (but in this special case it follows from the results of Section 9). Unfortunately, even the knowledge of this crossed-module structure is not enough to determine our Deligne-Mumford curve; but it is a step in the right direction. We address this issue in the subsequent sections by bringing some 2-group theory into the picture.

7.1. Graphs of groups as skeleta of open Deligne-Mumford curves. There is an alternative way of computing the homotopy groups of an open DeligneMumford curve, which is in a way simpler and more illuminating. The idea is that any open Deligne-Mumford curve has the homotopy type of a graph of groups in the sense of Serre [11]. We do not intend to give an overview of the theory of graphs of groups here, so we just confine ourselves to describing the procedure by which we associate a graph of groups to a Deligne-Mumford curve, and we leave it to the interested reader to verify that this approach will yield the same results (e.g., the same formula for the fundamental group etc.).

The idea is that one can retract an open Deligne-Mumford curve to a graph of groups. Assume $\mathcal{C}$ is a smooth Deligne-Mumford curve that is generically an $H$-gerbe, for some finite group $H$. Let $g$ be the genus of the underlying Riemann surface. Let $P_{1}, P_{2}, \cdots, P_{k}, k \geq 0$, be the orbifold points of $\mathcal{C}$, with their respective inertia groups $G_{1}, G_{2}, \cdots, G_{k}$, and let $Q_{1}, Q_{2}, \cdots, Q_{l}, l \geq 1$, be the punctured points.

Let us for one moment forget about the stacky structure of $\mathcal{C}$ and look at the underlying surface. This surface can be deformed into a bouquet of $2 g+l-1$ circles. If we take into account the stacky structure we should be careful how we treat the orbifold points $P_{1}, P_{2}, \cdots, P_{k}$. What we do is we choose a base point $P_{0}$, different from the $P_{i}$, and join $P_{0}$ to each orbifold point by a path (Figure 1). Now we perform the retraction of $\mathcal{C}$ in a way that all these paths are left intact during the retraction. The result is a graph of groups whose underlying graph consists of 

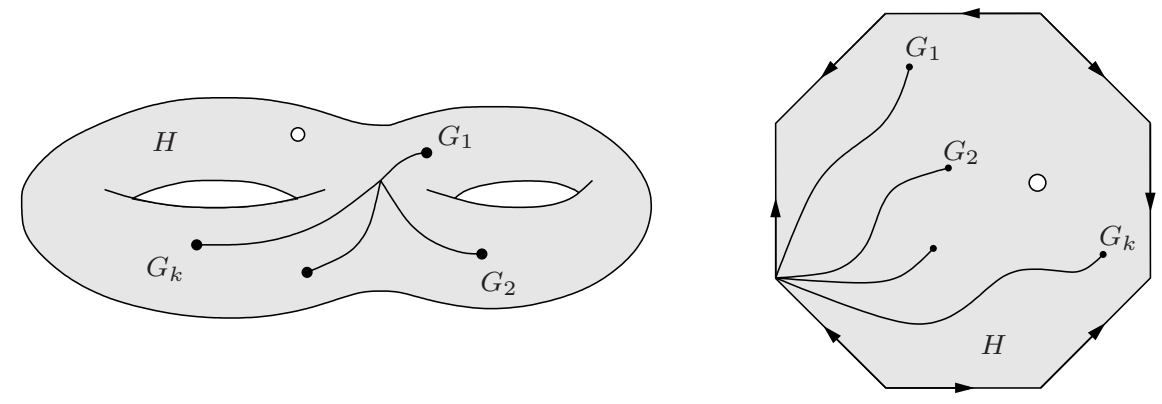

FiguRE 1

$2 g+l-1$ circles together with $k$ segments, all joined at a single point (Figure 2 ). The

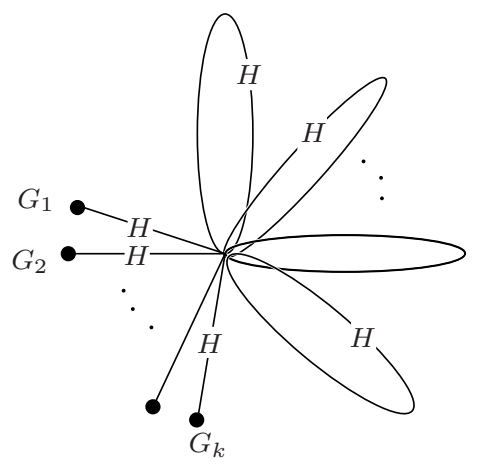

Figure 2

group associated to each edge is $H$, the group associated to $P_{0}$ is also $H$. The group associated to $P_{i}, i \geq 1$, is $G_{i}$. For every edge $P_{0} P_{i}$ The homomorphism $H \rightarrow G_{i}$ is just the natural inclusion (obtained from the path $P_{0} P_{i}$ ). For every circle in the bouquet, the homomorphism $H \rightarrow H$ is the one obtained from the monodromy action (see footnote in Section 4.2). This action determines a semi-direct product

$$
H \rtimes(\underset{2 g+l-1}{*} \mathbb{Z})
$$

which is the fundamental group of the bouquet. The fundamental group of the whole graph of groups is

$$
(H \rtimes(\underset{2 g+l-1}{*} \mathbb{Z})) *_{H}\left(*_{H} G_{i}\right) .
$$

In the case of a compact Deligne-Mumford curves one has to use 2-dimensional complexes of groups. The complexes of groups that arise in this way are of particularly simple form, because they can be obtained by attaching a simplex (with generic group $H$ ) to the complex of groups of an open Deligne-Mumford curves (which is homotopy equivalent to a graph of groups). This way we recover, for instance, the formula for the fundamental group of an arbitrary Deligne-Mumford curve (using van Kampen). 


\section{WeIGHTED PROJECTIVE GENERAL LINEAR 2-GROUPS}

In this section we invoke the full 2-categorical structure of the category of stacks, so our convention in Section 2 that all 2-isomorphic morphism are declared equal is no longer in effect.

As hinted in the previous section (see just after Proposition 7.6), crossed-modules (equivalently, 2-groups) appear naturally in the study of fundamental groups of Deligne-Mumford stacks. Even in the hyperbolic and Euclidean cases (Proposition 7.5) the 2-groups are implicitly present: $H \hookrightarrow \pi_{1} \mathcal{X}$ should be thought of as a crossed-module. The reason 2 -groups occur is that the automorphisms Aut $\mathcal{X}$ of a stack $\mathcal{X}$ (viewed as an object in the 2-category of stacks) naturally form a weak ${ }^{5}$ 2-group. A quick review of the basic facts of 2-group theory is given in Appendix.

Our main task in this section, and the next, is to study the 2-group of automorphisms of weighted projective stacks. Let $n_{1}, n_{2}, \cdots, n_{r}$ be a sequence of positive integers, and consider the weight $\left(n_{1}, n_{2}, \cdots, n_{r}\right)$ action of $\mathbb{C}^{*}$ on $\mathbb{C}^{r}-\{0\}$ (so $t \in \mathbb{C}^{*}$ acts as multiplication by $\left(t^{n_{1}}, t^{n_{2}}, \cdots, t^{n_{r}}\right)$ ). The stacky quotient of this action is the weighted projective stack $\mathcal{P}\left(n_{1}, n_{2}, \cdots, n_{r}\right)$. We define the weighted projective general linear 2-group $\operatorname{PGL}\left(n_{1}, n_{2}, \cdots, n_{r}\right)$ to be the 2 group associated to crossed-module $\left[\varphi: \mathbb{C}^{*} \rightarrow G_{n_{1}, n_{2}, \cdots, n_{r}}\right]$, where $G_{n_{1}, n_{2}, \cdots, n_{r}}$ is the group of all $\mathbb{C}^{*}$-equivariant (for the above weighted action) algebraic automorphisms $f: \mathbb{C}^{r}-\{0\} \rightarrow \mathbb{C}^{r}-\{0\}$. The homomorphism $\varphi: \mathbb{C}^{*} \rightarrow G_{n_{1}, n_{2}, \cdots, n_{r}}$ is the one induced from the $\mathbb{C}^{*}$-action. We take the action of $G_{n_{1}, n_{2}, \cdots, n_{r}}$ on $\mathbb{C}^{*}$ to be trivial.

Throughout this section we fix the weight sequence $n_{1}, n_{2}, \cdots, n_{r}$ and denote $G_{n_{1}, n_{2}, \cdots, n_{r}}$ simply by $G$.

Theorem 8.1. Let Aut ${ }^{a n} \mathcal{P}\left(n_{1}, n_{2}, \cdots, n_{r}\right)$ and Aut $\mathcal{P}\left(n_{1}, n_{2}, \cdots, n_{r}\right)$ be the 2groups of analytic and algebraic automorphisms of $\mathcal{P}\left(n_{1}, n_{2}, \cdots, n_{r}\right)$, respectively. Then,

i. The analyfication functor induces an equivalence

$$
\text { Aut } \mathcal{P}\left(n_{1}, n_{2}, \cdots, n_{r}\right) \rightarrow \operatorname{Aut}^{\text {an }} \mathcal{P}\left(n_{1}, n_{2}, \cdots, n_{r}\right)
$$

of 2-groups.

ii. The natural map

$$
\operatorname{PGL}\left(n_{1}, n_{2}, \cdots, n_{r}\right) \rightarrow \operatorname{Aut} \mathcal{P}\left(n_{1}, n_{2}, \cdots, n_{r}\right)
$$

is an equivalence of 2-groups.

First we prove a

Lemma 8.2. Let $H$ be an abelian group scheme acting on a connected scheme $X$ over $\mathbb{C}$, and let $X=[X / H]$ be the quotient stack. Let $G$ be the group of $H$ equivariant automorphism of $X$. Let Aut $X$ be the (weak) 2-group of automorphism of $X$.

i. The natural homomorphism $\varphi: H(\mathbb{C}) \rightarrow G(\mathbb{C})$ can be turned into a crossedmodule by taking the trivial action of $G$ on $H$.

ii. Let $\mathfrak{G}$ denote the 2-group associated to the crossed-module $[\varphi: H \rightarrow G]$. Then there is a natural map of 2-groups $\mathfrak{G} \rightarrow$ Aut $X$. Furthermore, this morphism induces an isomorphism on $\pi_{2}$.

\footnotetext{
${ }^{5}$ In fact, we still have the associativity on the nose. But inverses only exists in the weak sense.
} 
iii. Assume that $\mathcal{X}$ is a proper Deligne-Mumford stack and $H$ is affine. Then the induces map on $\pi_{1}$ is injective.

Proof. Part (i) is straightforward, because $\varphi$ maps $H$ into the center of $G$.

To prove part (ii), we recall the explicit description of the quotient stack $[X / H]$ :

$$
\begin{gathered}
\operatorname{Ob}[X / H](S)= \begin{cases}\left.(T, \alpha) \quad \mid \begin{array}{l}
T \text { an } H \text {-torsor over } S \\
\alpha: T \rightarrow X \text { an } H \text {-map }
\end{array}\right\} \\
\operatorname{Mor}[X / H](S)\left((T, \alpha),\left(T^{\prime}, \alpha^{\prime}\right)\right)=\left\{f: T \rightarrow T^{\prime} \text { an } H \text {-torsor map s.t. } \alpha^{\prime} \circ f=\alpha\right\}\end{cases}
\end{gathered}
$$

Any element of $g \in G(\mathbb{C})$ induces an automorphism of $\mathcal{X}$ (keep the same torsor $T$ and compose $\alpha$ with the action of $g$ on $X)$. Also, for any element $h \in H(\mathbb{C})$, there is a natural 2-isomorphism from the identity automorphism of $X$ to the automorphism induced by $\varphi(h) \in G(\mathbb{C})$ (which is by definition the same as the action of $h$ ). It is given by the multiplication action of $h^{-1}$ on the torsor $T$ (remember $H$ is abelian) which makes the following triangle commute

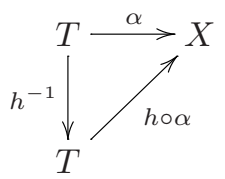

Interpreted in the language of 2-groups, this gives a morphism of 2-groups $\mathfrak{G} \rightarrow$ Aut $x$.

A general fact about stacks is that, the group of 2-isomorphisms from the identity automorphism of $X$ to itself is naturally isomorphic to the group of global sections of the inertia stack of $\mathcal{X}$. In the case $\mathcal{X}=[X / H]$, this is naturally isomorphic to the group of elements of $H(\mathbb{C})$ that act trivially on $X$. Note that this group is naturally isomorphic to $\pi_{2} \mathfrak{G}$. Therefore, the map $\mathfrak{G} \rightarrow$ Aut $\mathcal{X}$ induces an isomorphism on $\pi_{2}$. This completes the proof of (ii).

To prove part (iii) we need to show that, if the action of $g \in G(\mathbb{C})$ on $X$ is 2isomorphic to identity, then $g$ is in the image of $\varphi$. Let us fix such a 2-isomorphism. By looking at the groupoid $[X / H](X)$ and following around the effect of the action of $g$ and our 2-isomorphism on the point $X \rightarrow[X / H]$, we end up with an $H$-torsor map $F: H \times X \rightarrow H \times X$ making the following $H$-equivariant triangle commute:

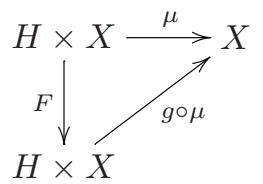

Here, $H \times X$ is the trivial $H$-torsor on $X$ and $\mu$ corresponds to the action of $H$ on $X$.

Composing $F$ with the identity section $X \rightarrow H \times X$, we obtain a map $f: X \rightarrow H$. It follows from the $H$-equivariance of the above diagram that, for any point $x$ in $X$, the effect of $g$ on $x$ is the same as the effect of $f(x)$ on $x$. In other words, $f(x) g^{-1}$ leaves $x$, or in fact every point $h(x)$ in the $H$-orbit of $x$, invariant. The same is true for $f(h(x)) g^{-1}$, for any $h \in H(\mathbb{C})$. This implies that, for any point $x$ of $X$, and any $h \in H(\mathbb{C})$, the element $r(x, h):=f(h(x)) f(x)^{-1}$ leaves $x$ (in fact the entire orbit of $x$ ) fixed; that is, $r(x, h)$ belongs to the stabilizer group $S_{x} \subseteq H$ of $x$. 
Therefore, the map $\rho: H \times X \rightarrow H \times X, \rho(h, x):=(r(h, x), x)$ factors through the stabilizer group scheme $S \rightarrow X$. Since $S \rightarrow X$ has discrete fibers, the restriction of $\rho$ to $H_{0} \times X$ factors through the identity section $\left(H_{0}\right.$ is the connected component of the identity). Hence, for every $h \in H_{0}(\mathbb{C})$ and $x \in X, r(h, x)$ is the identity. This implies that $f: X \rightarrow H$ is $H_{0}$-equivariant (for the trivial action of $H_{0}$ on $H$ ). So, we obtain an induced map $\left[X / H_{0}\right] \rightarrow H$. But we know that $\left[X / H_{0}\right]$, being finite over $[X / H]$, is proper and $H$ is affine, so this map must be constant; hence $f: X \rightarrow H$ is also constant. Let $h \in H(\mathbb{C})$ be the value of $f$. Then, the action of $h$ on $X$ coincides with the action of $g$. So $\varphi(h)=g$, which is what we wanted to prove.

Remark 8.3.

1. In fact $\operatorname{Aut}(X)$ is a 2 -group scheme and the above proof can be easily jazzed up to the level of 2-group schemes.

2. The assumption on schemes being over $\mathbb{C}$ is not necessary; we can work over any base scheme. Part (i) and (ii) of the lemma are quite formal and are valid in the analytic and topological settings as well. Part (iii) of the lemma is also valid in the analytic setting (but we have to require that $\left[X / H^{0}\right]$ is proper, where $H^{0}$ is the connected component of the identity).

3. Part (iii) of the lemma can be interpreted as saying that the morphism $\mathfrak{G} \rightarrow$ Aut $X$ is "injective". That is, its kernel is equivalent to the trivial 2-group.

Proof of Theorem 8.1. Part (i) is a GAGA type statement and follows from Theorem 1.1 of [5]. To prove part (ii) we may assume $r \geq 2$. By the previous lemma, the map

$$
P G L\left(n_{1}, n_{2}, \cdots, n_{r}\right) \rightarrow \operatorname{Aut} \mathcal{P}\left(n_{1}, n_{2}, \cdots, n_{r}\right)
$$

induces an isomorphism on $\pi_{2}$ and an injection on $\pi_{1}$. So we only need to prove the surjectivity on $\pi_{1}$. First, we show that for any automorphism $f: \mathcal{P}\left(n_{1}, n_{2}, \cdots, n_{r}\right) \rightarrow$ $\mathcal{P}\left(n_{1}, n_{2}, \cdots, n_{r}\right)$ there is a lift $F: \mathbb{C}^{r}-\{0\} \rightarrow \mathbb{C}^{r}-\{0\}$ making the following diagram 2-cartesian

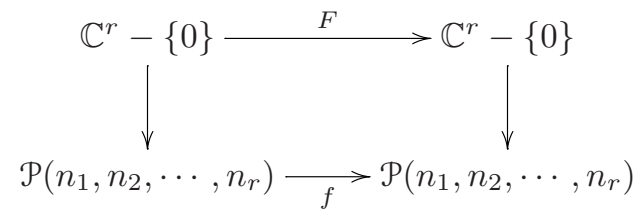

Recall that the map $\mathbb{C}^{r}-\{0\} \rightarrow \operatorname{Pic} \mathcal{P}\left(n_{1}, n_{2}, \cdots, n_{r}\right)$ is the total space of the Hopf bundle $L \in \operatorname{Pic} \mathcal{P}\left(n_{1}, n_{2}, \cdots, n_{r}\right)$ with the identity section removed. We know that Pic $\mathcal{P}\left(n_{1}, n_{2}, \cdots, n_{r}\right) \cong \mathbb{Z}$, with the Hopf bundle corresponding to $1 \in \mathbb{Z}$. Any automorphism induces a trivial action on $\operatorname{Pic} \mathcal{P}\left(n_{1}, n_{2}, \cdots, n_{r}\right)$ (i.e. it preserves the "degree"). Hence, $f^{*} L \cong L$. A choice of an isomorphism $f^{*} L \stackrel{\sim}{\longrightarrow}$ gives rise to the desired 2-cartesian diagram.

If we show that $F$ is $\mathbb{C}^{*}$-equivariant we are done. Since the above diagram is 2-cartesian, there is a groupoid map from $\mathbb{C}^{*} \times \mathbb{C}^{r}-\{0\} \rightrightarrows \mathbb{C}^{r}-\{0\}$ to itself whose corresponding map on the 'objects-space' is $F: \mathbb{C}^{r}-\{0\} \rightarrow \mathbb{C}^{r}-\{0\}$. Let us denote the map on the 'arrow space' by $\Phi: \mathbb{C}^{*} \times \mathbb{C}^{r}-\{0\} \rightarrow \mathbb{C}^{*} \times \mathbb{C}^{r}-\{0\}$. Since 
$\Phi$ commutes with the source map (projection) $\mathbb{C}^{*} \times \mathbb{C}^{r}-\{0\} \rightarrow \mathbb{C}^{r}-\{0\}$, for any $x \in \mathbb{C}^{r}-\{0\}$ we obtain a map $\Phi_{x}: \mathbb{C}^{*} \rightarrow \mathbb{C}^{*}$ (the first $\mathbb{C}^{*}$ is the fiber over $x$ and the second is the fiber over $F(x)$ ). A priori, the $\Phi_{x}$ are not group homomorphisms, but since $\Phi$ sends the identity to identity, so do $\Phi_{x}$. It is easy to show that the only algebraic (or analytic) isomorphisms $\mathbb{C}^{*} \rightarrow \mathbb{C}^{*}$ that preserve 1 are the identity map $x \mapsto x$ and the inversion map $x \mapsto \frac{1}{x}$. So each $\Phi_{x}: \mathbb{C}^{*} \rightarrow \mathbb{C}^{*}$ is either identity or inversion. By continuity, either all $\Phi_{x}$ are identity, or all inversions. In the first case, it follows that $F$ is $\mathbb{C}^{*}$-equivariant, which is what we want. In the second case, it follows that $F$ is twisted $\mathbb{C}^{*}$-equivariant; that is, for every $a \in \mathbb{C}^{*}$, and every $x \in \mathbb{C}^{r}-\{0\}$, we have $F(a \cdot x)=\frac{1}{a} \cdot x$. We show that this is impossible. First observe that, since $r \geq 2$, the map $F: \mathbb{C}^{r}-\{0\} \rightarrow \mathbb{C}^{r}-\{0\}$ extends to $\mathbb{C}^{r}$. Consider the point $(1,0, \cdots, 0) \in \mathbb{C}^{r}-\{0\}$. If $F$ is twisted $\mathbb{C}^{*}$-equivariant, we have $F\left(a^{n_{1}}, 0, \cdots, 0\right)=a^{-n_{1}} F(1,0, \cdots, 0)$, for every $a \in \mathbb{C}^{*}$. Letting $a$ approach zero, we see that the left hand side approaches to $F(0,0, \cdots, 0)$, which is a well-defined point, but the right hand side goes to infinity. This contradiction shows that $F$ can not be twisted $\mathbb{C}^{*}$-equivariant, so it has to be $\mathbb{C}^{*}$-equivariant. The proof is now complete.

Proposition 8.4. Let $n_{1}, n_{2}, \cdots, n_{r}$ be a sequence of positive integers, and let d be their greatest common divisor. Then, the weighed projective stack $\mathcal{P}\left(n_{1}, n_{2}, \cdots, n_{r}\right)$

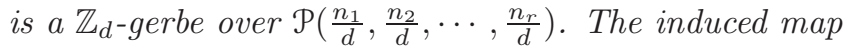

$$
\operatorname{PGL}\left(n_{1}, n_{2}, \cdots, n_{r}\right) \rightarrow \operatorname{PGL}\left(\frac{n_{1}}{d}, \frac{n_{2}}{d}, \cdots, \frac{n_{r}}{d}\right)
$$

of automorphism 2-groups (obtained from the functoriality of the "underlying orbifold" construction, see Proposition 4.6) induces an isomorphism on $\pi_{1}$. That is,

$$
\pi_{1} \operatorname{PGL}\left(n_{1}, n_{2}, \cdots, n_{r}\right) \stackrel{\sim}{\longrightarrow} \pi_{1} \operatorname{PGL}\left(\frac{n_{1}}{d}, \frac{n_{2}}{d}, \cdots, \frac{n_{r}}{d}\right)
$$

Note that $\operatorname{PGL}\left(\frac{n_{1}}{d}, \frac{n_{2}}{d}, \cdots, \frac{n_{r}}{d}\right)$ is equivalent to a group, that is,

$$
\operatorname{PGL}\left(\frac{n_{1}}{d}, \frac{n_{2}}{d}, \cdots, \frac{n_{r}}{d}\right) \stackrel{\sim}{\longrightarrow} \pi_{1} \operatorname{PGL}\left(\frac{n_{1}}{d}, \frac{n_{2}}{d}, \cdots, \frac{n_{r}}{d}\right) .
$$

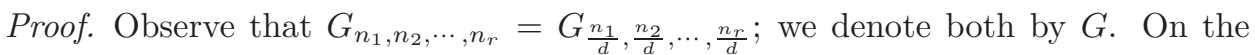
level of crossed-modules, our 2-group morphism look as follows

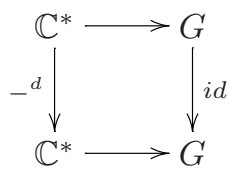

(see just before Theorem 8.1 for notation). The result is obvious.

8.1. Explicit description of $\operatorname{PGL}(m, n)$. The crossed-module $\operatorname{PGL}(m, n)$ can be described quite explicitly. To do so, we first have to determine $G_{m, n}$. We do this by considering three cases. As before, we denote $G_{m, n}$ simply by $G$.

The case $m<n, m \nmid n$.

It is easy to see that an element in $G$ is a map of the form

$$
(x, y) \mapsto\left(\lambda_{1} x, \lambda_{2} y\right),
$$


for some fix $\lambda_{1}, \lambda_{2} \in \mathbb{C}^{*}$. So we have $G \cong \mathbb{C}^{*} \times \mathbb{C}^{*}$. The map $\varphi$ : $\mathbb{C}^{*} \rightarrow G$ is given by

$$
\lambda \mapsto\left(\lambda^{m}, \lambda^{n}\right) .
$$

The map

$$
\begin{aligned}
G \cong \mathbb{C}^{*} \times \mathbb{C}^{*} & \rightarrow \mathbb{C}^{*} \\
\left(\lambda_{1}, \lambda_{2}\right) & \mapsto \lambda_{1}^{\frac{n}{d}} \lambda_{2}^{-\frac{m}{d}}
\end{aligned}
$$

induces an isomorphism $\pi_{1} \operatorname{PGL}(m, n) \cong \mathbb{C}^{*}$. We can produce a section

$$
\sigma: \pi_{1} \operatorname{PGL}(m, n) \rightarrow G
$$

for this map by choosing integers $r, s$ such that $s m+r n=d$ and setting $\sigma(\lambda)=$ $\left(\lambda^{-r}, \lambda^{s}\right)$.

Convention. Throughout the paper, given $m, n$, we fix a pair $r, s$ with $s m+r n=d$. When $m$ divides $n$ we take $s=1$ and $r=0$.

The case $m<n, m \mid n$.

Every element in $G$ is a map of the form

$$
(x, y) \mapsto\left(\lambda_{1} x, \lambda_{2} y+a x^{\frac{n}{m}}\right),
$$

for some $\lambda_{1}, \lambda_{2} \in \mathbb{C}^{*}$ and $a \in \mathbb{C}$. So we have

$$
G \cong\left(\mathbb{C}^{*} \times \mathbb{C}^{*}\right) \ltimes \mathbb{C},
$$

where the action of an element $\left(\lambda_{1}, \lambda_{2}\right) \in \mathbb{C}^{*} \times \mathbb{C}^{*}$ on an element $a \in \mathbb{C}$ is given by

$$
\left(\lambda_{1}, \lambda_{2}\right) \cdot a=\lambda_{1}^{-\frac{n}{m}} \lambda_{2} a .
$$

(Note the similarity with the group of $2 \times 2$ lower-triangular matrices.)

The map $\varphi: \mathbb{C}^{*} \rightarrow G$ is given by

$$
\lambda \mapsto\left(\lambda^{m}, \lambda^{n}, 0\right) .
$$

The map

$$
\begin{aligned}
G \cong\left(\mathbb{C}^{*} \times \mathbb{C}^{*}\right) \ltimes \mathbb{C} & \rightarrow \mathbb{C}^{*} \ltimes \mathbb{C} \\
\left(\lambda_{1}, \lambda_{2}, a\right) & \mapsto\left(\lambda_{1}^{-\frac{n}{m}} \lambda_{2}, a\right)
\end{aligned}
$$

induces an isomorphism $\pi_{1} \operatorname{PGL}(m, n) \cong \mathbb{C}^{*} \ltimes \mathbb{C}$. In the latter semi-direct product the action of $\mathbb{C}^{*}$ is simply the multiplication action.

There is a section $\sigma: \pi_{1} \operatorname{PGL}(m, n) \rightarrow G$ to the above map which is given by $\sigma(\lambda, a)=(1, \lambda, a)$.

The case $m=n$.

We switch the notation from $m=n$ to $d$. In this case we obviously have $G \cong \mathrm{GL}_{2}$. The map $\varphi: \mathbb{C}^{*} \rightarrow G$ is given by

$$
\lambda \mapsto\left(\begin{array}{cc}
\lambda^{d} & 0 \\
0 & \lambda^{d}
\end{array}\right)
$$

We summarize the above discussion in the following propositions. 
Proposition 8.5. Let $m, n$ be distinct positive integers, and let $d=\operatorname{gcd}(m, n)$. Then $\operatorname{PGL}(m, n)$ is a split 2-group. In particular, it is classified by its homotopy groups:

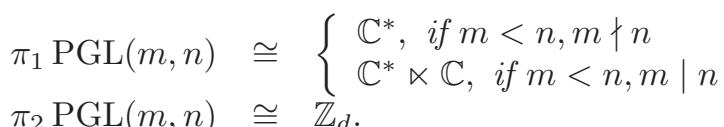

(In the case $m \mid n$, the action of $\mathbb{C}^{*}$ on $\mathbb{C}$ in the semi-direct product $\mathbb{C}^{*} \ltimes \mathbb{C}$ is simply the multiplication action.)

Proposition 8.6. The 2-group $\mathrm{PGL}(d, d)$ is given by the following crossed-module:

$$
\left[\alpha: \mathbb{C}^{*} \stackrel{\left(\lambda^{d}, \lambda^{d}\right)}{\longrightarrow} \mathrm{GL}_{2}\right] .
$$

We have $\pi_{1} \operatorname{PGL}(d, d) \cong \mathrm{PGL}_{2}$ and $\pi_{2} \operatorname{PGL}(d, d) \cong \mathbb{Z}_{d}$.

\section{Classification of spherical Deligne-Mumford curves}

Having described the structure of the 2-group of automorphisms of a weighted projective line $\mathcal{P}(m, n)$, we can now classify spherical Deligne-Mumford curves.

Let $X$ be a spherical Deligne-Mumford curve. We know that the universal cover of $X$ is a weighted projective line $\mathcal{P}(m, n)$ (Proposition 7.2). The deck transformations of the covering map $\mathcal{P}(m, n) \rightarrow X$ give us a homotopy class of map of 2-groups $\pi_{1} X \rightarrow \operatorname{PGL}(m, n)$ that is unique up to conjugation. Conversely, given such a conjugacy class, we can recover $\mathcal{X}$ up to isomorphism [7]. So, what we have to do is to classify conjugacy classes of (homotopy classes of) maps $\pi_{1} X \rightarrow \operatorname{PGL}(m, n)$.

Before presenting the classification result, let us explain how this 2-group theoretic approach fits with the results of Section 7 . We show how to derive the exact sequence of Proposition 7.6 from the map $\pi_{1} X \rightarrow \operatorname{PGL}(m, n)$. (Note: the numbers $m$ and $n$ here are different from those of Proposition 7.6.)

By Proposition 7.6, the universal cover of $\mathcal{C}$ is $\mathcal{P}\left(\frac{m}{d}, \frac{n}{d}\right)$, where $d=(m, n)$. Let $\mathfrak{P}=\pi_{1} \mathcal{X}$, viewed as a 2-group, and let $\mathfrak{H}$ be the kernel of the map $\mathfrak{P} \rightarrow \operatorname{PGL}(m, n)$. After passing to classifying spaces and taking homotopy groups, we obtain the following fiber homotopy exact sequence:

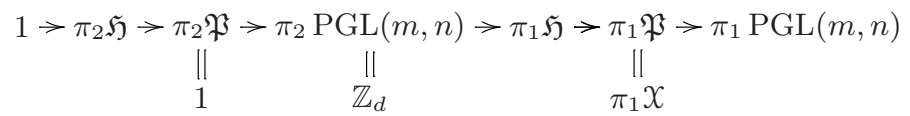

It follows from the above exact sequence that $\pi_{2} \mathfrak{H}$ is trivial. By Proposition 8.4, we have $\pi_{1} \operatorname{PGL}(m, n) \cong \operatorname{PGL}\left(\frac{m}{d}, \frac{n}{d}\right)$. Via this isomorphism, the image of $\pi_{1} X$ is identified with $\pi_{1} \mathrm{C} \subset \operatorname{PGL}\left(\frac{m}{d}, \frac{n}{d}\right)$. So the above exact sequence takes the following form:

$$
1 \rightarrow \mathbb{Z}_{d} \rightarrow \pi_{1} \mathfrak{H} \rightarrow \pi_{1} X \rightarrow \pi_{1} \mathcal{C} \rightarrow 1 .
$$

It is easy to guess what $\pi_{1} \mathfrak{H}$ is; it is simply $H$, and the above exact sequence is isomorphic to that of Proposition 7.6.

Now we get back to the classification of spherical Deligne-Mumford curves. To do so, we combine Theorem 10.3 of the Appendix with the results of the preceding sections to classify conjugacy classes of homotopy classes of maps $\Gamma \rightarrow \operatorname{PGL}(m, n)$ for any discrete group $\Gamma$. As a byproduct of this classification, we obtain an explicit (and canonical) description of a spherical Deligne-Mumford curve as a quotient of $\mathbb{C}^{2}-\{0\}$. 
We consider two cases.

9.1. Universal cover $\mathcal{P}(m, n), m \neq n$. Let $d=\operatorname{gcd}(m, n)$. We saw in Section 8.1 that $\operatorname{PGL}(m, n)$ is split in this case (Proposition 8.5). We fix the splitting as in Section 8.1. This immediately implies the following proposition.

Proposition 9.1. For any group $\Gamma$, there is a natural bijection

$$
\operatorname{Hom}_{\operatorname{Ho}(2 G p)}(\Gamma, \operatorname{PGL}(m, n)) \cong \begin{cases}\operatorname{Hom}\left(\Gamma, \mathbb{C}^{*}\right) \times H^{2}\left(\Gamma, \mu_{d}\right) & \text { if } m \nmid n \\ \operatorname{Hom}\left(\Gamma, \mathbb{C}^{*} \ltimes \mathbb{C}\right) \times H^{2}\left(\Gamma, \mu_{m}\right) & \text { if } m \mid n\end{cases}
$$

where the the cohomology groups are with trivial coefficients and the semi-direct product is formed from the standard action of $\mathbb{C}^{*}$ on $\mathbb{C}$.

Proof. Follows from Corollary 10.6 of Appendix.

To characterize Deligne-Mumford curves $\mathcal{X}$ with fundamental group $\Gamma$ and universal cover $\mathcal{P}(m, n)$, what we need is to classify conjugacy classes of elements in $\operatorname{Hom}_{\mathrm{Ho}(2 G p)}(\Gamma, \mathrm{PGL}(m, n))$. First we prove a lemma.

Lemma 9.2. For every group homomorphism $\psi: \Gamma \rightarrow \mathbb{C}^{*} \ltimes \mathbb{C}$ there is a unique character $\chi: \Gamma \rightarrow \mathbb{C}^{*}$ that is conjugate to $\psi$. (Here we think of $\mathbb{C}^{*}$ as the first component of $\mathbb{C}^{*} \ltimes \mathbb{C}$.)

Proof. Every group homomorphism $\psi: \Gamma \rightarrow \mathbb{C}^{*} \ltimes \mathbb{C}$ is of the form $(\chi, \eta)$, where $\chi$ is a character of $\Gamma$ and $\eta(u)=a(1-\chi(u))$ for some $a \in \mathbb{C}$. (If we think of $\mathbb{C}^{*} \ltimes \mathbb{C}$ as the group of affine transformations of $\mathbb{C}$, this is saying that the action of $\psi$ is rotation around the point $a \in \mathbb{C}$, via the character $\chi$.) Using the element $(1, a) \in \mathbb{C}^{*} \ltimes \mathbb{C}$ we can conjugate this with $\chi$ itself (in other words, we are sending $a$ to the origin).

Proposition 9.3. Assume $m \neq n$. Then the conjugacy classes of homotopy classes of maps from $\Gamma$ to $\mathrm{PGL}(m, n)$ are in bijection with

$$
\operatorname{Hom}\left(\Gamma, \mathbb{C}^{*}\right) \times H^{2}\left(\Gamma, \mu_{d}\right),
$$

where $d=\operatorname{gcd}(m, n)$.

Proof. If non of $m$ and $n$ divides the other, every two conjugate elements in $H_{\text {om }}{ }_{\mathrm{Ho}(2 G p)}(\Gamma, \operatorname{PGL}(m, n))$ are in fact equal.

When $m$ divides $n$, we know that $\pi_{1} \operatorname{PGL}(m, n) \cong \mathbb{C}^{*} \ltimes \mathbb{C}$. Lemma 9.2 implies that every conjugacy class in

$$
\operatorname{Hom}_{\operatorname{Ho}(\mathbf{2 G p})}(\Gamma, \operatorname{PGL}(m, n)) \cong \operatorname{Hom}\left(\Gamma, \mathbb{C}^{*} \ltimes \mathbb{C}\right) \times H^{2}\left(\Gamma, \mu_{m}\right)
$$

can be conjugated to an element $(\chi, c)$ in

$$
\operatorname{Hom}\left(\Gamma, \mathbb{C}^{*}\right) \times H^{2}\left(\Gamma, \mu_{m}\right) \subseteq \operatorname{Hom}\left(\Gamma, \mathbb{C}^{*} \ltimes \mathbb{C}\right) \times H^{2}\left(\Gamma, \mu_{m}\right) .
$$

It also implies that $\chi$ is unique. So, we need to show that, if $(\chi, c)$ and $\left(\chi, c^{\prime}\right)$ are conjugate, then $c=c^{\prime}$. In other words, we have to show that, for every $a \in G_{m, n}$ such that $c_{\bar{a}} \circ \chi=\chi: \Gamma \rightarrow \pi_{1} \mathfrak{G}$, the induced conjugation action

$$
a_{*}: \operatorname{Hom}_{\mathrm{Ho}(\mathbf{2 G p})}(\Gamma, \operatorname{PGL}(m, n))_{\chi} \rightarrow \operatorname{Hom}_{\mathrm{Ho}(\mathbf{2 G})}(\Gamma, \operatorname{PGL}(m, n))_{\chi}
$$

is trivial. (Note that $a_{*}$ is not a priori a group homomorphisms.)

By Corollary 10.9 of Appendix, it is enough to show that $a_{*}$ has a fixed point. To obtain such a fixed point, consider the splitting $\pi_{1} \operatorname{PGL}(m, n) \rightarrow G_{m, n}$, and use it to lift $\chi$ to $\tilde{\chi}: \Gamma \rightarrow G_{m, n}$ We claim that $\tilde{\chi}$ is a fixed point of $a_{*}$. 
We may assume that $\chi$ is non-trivial. Notice that $\bar{a} \in \pi_{1} \operatorname{PGL}(m, n) \cong \mathbb{C}^{*} \ltimes \mathbb{C}$ commutes with every element in the image of $\chi$. It follows that $\bar{a} \in \mathbb{C}^{*} \subset \mathbb{C}^{*} \ltimes \mathbb{C}$. Therefore, $a$ is in the inverse image of $\mathbb{C}^{*} \in G_{m, n}$. This inverse image is isomorphic to $\mathbb{C}^{*} \times \mathbb{C}^{*}$, which is abelian. Since the image of $\tilde{\chi}$ also lands in this abelian subgroup, conjugation by a leaves $\tilde{\chi}$ invariant, which is what we wanted to prove.

By Proposition 9.3, every Deligne-Mumford curve $\mathcal{X}$ with universal cover $\mathcal{P}(m, n)$ such that $\pi_{1} X \cong \Gamma$ gives rise to an element in $\operatorname{Hom}\left(\Gamma, \mathbb{C}^{*}\right) \times H^{2}\left(\Gamma, \mu_{d}\right)$. Of course this element depends on the choice of the isomorphism $\pi_{1} x \stackrel{\sim}{\longrightarrow}$, so we have a bijection between the set of isomorphism classes of Deligne-Mumford curves whose fundamental groups is isomorphic to $\Gamma$ and the set

$$
\left(\operatorname{Hom}\left(\Gamma, \mathbb{C}^{*}\right) \times H^{2}\left(\Gamma, \mu_{d}\right)\right) / \operatorname{Aut}(\Gamma)
$$

Taking into account that elements in $H^{2}\left(\Gamma, \mu_{d}\right)$ can be thought of as (isomorphisms classes of) central extensions of $\Gamma$ by $\mu_{d}$, this can be rephrased as follows.

Theorem 9.4. Let $m \neq n$ be given positive integer numbers, and let $d=\operatorname{gcd}(m, n)$. Then, the isomorphism classes of Deligne-Mumford curves $\mathcal{X}$ with universal cover $\mathcal{P}(m, n)$ are in natural bijection with isomorphism classes of pairs $(K, \chi)$, where $K$ is a finite group containing $\mu_{d}$ as a central subgroup and $\chi: K / \mu_{d} \rightarrow \mathbb{C}^{*}$ is a character.

Another way to reformulate Proposition 9.3 is as follows. Let $G_{m, n}^{\prime} \subset G_{m, n}$ be the subgroup $\mathbb{C}^{*} \times \mathbb{C}^{*}$. Form the sub-crossed-module $\operatorname{PGL}(m, n)^{\prime}=\left[\varphi: \mathbb{C}^{*} \rightarrow\right.$ $\left.G_{m, n}^{\prime}\right]$. Proposition 9.3 is then equivalent to the following.

Proposition 9.5. Every conjugacy class in $\operatorname{Hom}_{\mathrm{Ho}(\mathbf{2 G})}(\Gamma, \operatorname{PGL}(m, n))$ has a unique representative in $\operatorname{Hom}_{\mathrm{Ho}(\mathbf{2} \mathbf{G p})}\left(\Gamma, \operatorname{PGL}(m, n)^{\prime}\right)$. In other words, the set of conjugacy classes of elements in $\operatorname{Hom}_{\mathrm{Ho}(\mathbf{2 G p})}(\Gamma, \operatorname{PGL}(m, n))$ is in natural bijection with $\operatorname{Hom}_{\mathrm{Ho}(\mathbf{2 G p})}\left(\Gamma, \operatorname{PGL}(m, n)^{\prime}\right)$.

Corollary 9.6. All Deligne-Mumford curves whose universal cover is $\mathcal{P}(m, n)$ are given by triples $(\iota, E, \rho)$, where

i. $\iota: \mathbb{C}^{*} \hookrightarrow E$ is a central injection,

ii. $\rho: E \rightarrow \mathbb{C}^{*} \times \mathbb{C}^{*}$ is a group homomorphism,

such that the following two conditions are satisfied:

a. $\rho \circ \iota: \mathbb{C}^{*} \rightarrow \mathbb{C}^{*} \times \mathbb{C}^{*}$ is the diagonal $(m, n)$-th power map,

b. $E / \mathbb{C}^{*}$ is finite.

The curves given by $(\iota, E, \rho)$ and $\left(\iota^{\prime}, E^{\prime}, \rho^{\prime}\right)$ are isomorphic if and only if there is an isomorphism $f: E \rightarrow E^{\prime}$ such that $\rho^{\prime} \circ f=\rho$.

Proof. In view of Proposition 9.5, this is just a special case of Theorem 10.3 of Appendix, with $\mathfrak{G}=\operatorname{PGL}(m, n)^{\prime}$.

Let us describe how from a given pair $(K, \chi)$ as in Theorem 9.4 we can reconstruct $X$. What we will actually do is to produce a triple $(\iota, E, \rho)$ as in Corollary 9.6; we will be implicitly using Corollary 10.8 of Appendix. Let $E:=\mathbb{C}^{*} \times{ }_{\mu_{d}} K$ be the push out of $K$ along the inclusion map $\mu_{d} \rightarrow \mathbb{C}^{*}$. Let $r$ and $s$ be integers such that $s m+r n=d$ (as in Section 8.1). Extend the weighted action of $\mathbb{C}^{*}$ on $\mathbb{C}^{2}$ to $E$ by sending $(\lambda, u) \in E$ to the diagonal matrix

$$
\left(\begin{array}{cc}
\chi(u)^{-r} \lambda^{m} & 0 \\
0 & \chi(u)^{s} \lambda^{n}
\end{array}\right)
$$


(This corresponds to the map $\rho$ of Corollary 9.6.) Then, $X \cong\left[\mathbb{C}^{2}-\{0\} / E\right]$. This is an $H$-gerbe over $\mathcal{C}=\left[\mathcal{P}\left(\frac{m}{d}, \frac{n}{d}\right) / \chi(K)\right]$, where $\chi(K) \subset \mathbb{C}^{*}$ is acting by rotations around the origin, and $H=\operatorname{ker} \chi$. The fundamental group of $X$ is isomorphic to $K / \mu_{d}$.

To get a better picture, let us complete the exact sequence of Proposition 7.6.i as follows:

$$
\begin{gathered}
K \\
\uparrow \searrow \\
1 \rightarrow \mu_{d} \rightarrow \pi_{1} X \rightarrow \pi_{1} \mathrm{e} \rightarrow 1 .
\end{gathered}
$$

9.2. Universal cover $\mathcal{P}(d, d)$. This case is more complicated than the previous one since $\operatorname{PGL}(d, d)$ is no longer split. As a consequence, the classification result is less explicit. The number $d$ is fixed throughout this section.

Proposition 9.7. For any discrete group $\Gamma$ there is a natural bijection between $H_{\mathrm{Hom}}^{\mathrm{Ho}(2 G p)}(\Gamma, P G L(d, d))$ and the set of all isomorphism classes of diagrams

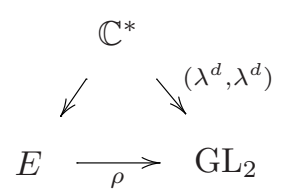

Here $E$ is a central extension of $\Gamma$ by $\mathbb{C}^{*}, \rho$ is a $\mathbb{C}$-representation of $E$ and the triangle commutes. (Isomorphism has the obvious meaning; see Theorem 10.3.)

Proof. This follows from Theorem 10.3 of Appendix.

Thus, every pair $(E, \rho)$ defines a Deligne-Mumford curve with universal cover $\mathcal{P}(d, d)$. Conversely, every such Deligne-Mumford curve defines a pair $(E, \rho)$, that is unique up to conjugation (this means, for any other $\left(E^{\prime}, \rho^{\prime}\right)$ there is an isomorphism $f: E \rightarrow E^{\prime}$ and an element $a \in G L_{2}$ such that $\rho^{\prime} \circ f=c_{a} \circ \rho$, where $c_{a}: G L_{2} \rightarrow G L_{2}$ is conjugation by $a$ ).

Let us explain how this correspondence works. Note that given $(E, \rho)$, taking $\pi_{1}$ gives a homomorphism of groups $\Gamma \rightarrow P G L_{2}$. Let $\bar{\Gamma}$ denote the image. (Of course, $\bar{\Gamma}$ has to be 'platonic'.) Then, the Deligne-Mumford curve corresponding to $(E, \rho)$ is

$$
X=\left[\mathbb{C}^{2}-\{0\} / E\right] .
$$

It is an $H$-gerbe over the orbifold $\mathcal{C}=\left[\mathbb{P}^{1} / \bar{\Gamma}\right]$, where $H=\operatorname{ker} \rho$. Moreover, we have $\pi_{1} X=\Gamma$ and $\mathcal{X}=[\mathcal{P}(d, d) / \Gamma]$.

The Deligne-Mumford curve, its orbifold and their universal covers fit in the following commutative diagram:

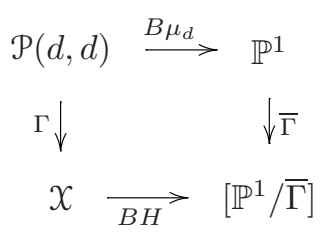

(The arrows are labelled with their fibers.) 
Corollary 9.8. All Deligne-Mumford curves whose universal cover is $\mathcal{P}(d, d)$ are given by triples $(\iota, E, \rho)$, where

i. $\iota: \mathbb{C}^{*} \hookrightarrow E$ is a central injection,

ii. $\rho: E \rightarrow G L_{2}$ is a 2-dimensional representation,

such that the following two conditions are satisfied:

a. $\rho \circ \iota: \mathbb{C}^{*} \rightarrow G L_{2}$ is the diagonal $(d, d)$-th power map,

b. $E / \mathbb{C}^{*}$ is finite.

The curves given by $(\iota, E, \rho)$ and $\left(\iota^{\prime}, E^{\prime}, \rho^{\prime}\right)$ are isomorphic if and only if there is an isomorphism $f: E \rightarrow E^{\prime}$ and an element $a \in G L_{2}$ such that $\rho^{\prime} \circ f=c_{a} \circ \rho$, where $c_{a}: G L_{2} \rightarrow G L_{2}$ is conjugation by $a$.

The last part of the above corollary is simply saying that the curves given by $(\iota, E, \rho)$ and $\left(\iota^{\prime}, E^{\prime}, \rho^{\prime}\right)$ are isomorphic if and only if the corresponding maps in $\operatorname{Hom}_{\mathrm{Ho}(\mathbf{2} \mathbf{G p})}(\Gamma, \mathrm{PGL}(d, d)$ are conjugate (see page 38$)$. This is unfortunately not as satisfactory as Corollary 9.6, because there, thanks to Proposition 9.3, we have a recognition principle for conjugacy classes in in $\operatorname{Hom}_{\mathrm{Ho}(\mathbf{2} \mathbf{G p})}(\Gamma, \mathrm{PGL}(m, n)$; in fact, Proposition 9.3 gives us a canonical representative for each conjugacy class. We do not have such a clean picture in the case of $\operatorname{PGL}(d, d)$, making it more difficult to decide whether given two triples $(\iota, E, \rho)$ and $\left(\iota^{\prime}, E^{\prime}, \rho^{\prime}\right)$ are conjugate or not. We address this issue in more detail in the next subsection.

9.3. Conjugacy classes in $\operatorname{Hom}_{\mathrm{Ho}(\mathbf{2} \mathbf{G p})}(\Gamma, \mathrm{PGL}(d, d))$. We describe conjugacy classes of elements in $\operatorname{Hom}_{\mathrm{Ho}(\mathbf{2 G p})}(\Gamma, \mathrm{PGL}(d, d))$. We do our analysis by fixing a group homomorphism $\chi: \Gamma \rightarrow \mathrm{PGL}_{2}$ and studying the conjugacy classes in $\operatorname{Hom}_{\mathrm{Ho}(\mathbf{2} \mathbf{G p})}(\Gamma, \mathrm{PGL}(d, d))_{\chi}$. We will assume throughout this section that this set is non-empty.

It turns out that conjugacy classes have length 1,2 or 4 , but most of the time 1 . We begin with an easy proposition.

Proposition 9.9. Let $\chi: \Gamma \rightarrow \mathrm{PGL}_{2}$ be a group homomorphisms, and let $I \subset$ $P G L_{2}$ be its image. Let $C$ be the centralizer of $I$ in $\mathrm{PGL}_{2}$. Consider the action of $C$ on $\operatorname{Hom}_{\mathrm{Ho}(\mathbf{2} \mathbf{G p})}(\Gamma, \operatorname{PGL}(d, d))_{\chi}$ in which $a \in C$ acts by $c_{\tilde{a}}$, where $\tilde{a} \in \mathrm{GL}_{2}$ is an arbitrary lift of a. (Note that this is independent of the choice of the lift $\tilde{a}$.) Then, the orbits of this action are precisely the conjugacy classes of elements in $\operatorname{Hom}_{\mathrm{Ho}(\mathbf{2} \mathbf{G p})}(\Gamma, \mathrm{PGL}(d, d))_{\chi}$.

Proof. Obvious.

To classify conjugacy classes of elements in $\operatorname{Hom}_{\mathrm{Ho}(\mathbf{2} \mathbf{G p})}(\Gamma, \operatorname{PGL}(d, d))_{\chi}$ we consider two cases: the image $I$ of $\chi$ is dihedral or not. The latter case is easier.

Proposition 9.10. Assume that the image $I \subset \mathrm{PGL}_{2}$ of $\chi$ is not dihedral. Then, every two conjugate elements in $\operatorname{Hom}_{\mathrm{Ho}(\mathbf{2} \mathbf{G p})}(\Gamma, \mathrm{PGL}(d, d))_{\chi}$ are actually equal.

Proof. If $I$ is not cyclic, a case by case inspection shows that the centralizer of $I$ in $P G L_{2}$ is trivial. So the claim is true in these cases. In the case where $I$ is cyclic, we may assume that $I$ is a group of rotations around the origin. In this case, the centralizer of $I$ in $\mathrm{PGL}_{2}$ is the entire group $\mathbb{C}^{*}$ of rotations around the origin. The inverse image of this group in $\mathrm{GL}_{2}$ is isomorphic to $\mathbb{C}^{*} \times \mathbb{C}^{*}$, which is abelian. So the claim is also true in this case.

In the rest of this section we assume that $I$ is dihedral. 
Proposition 9.11. Assume $I$ is dihedral, say $I \cong D_{n}=\mu_{n} \rtimes \mathbb{Z}_{2}$. Let $C$ be the centralizer of I in $\mathrm{PGL}_{2}$. We have the following possibilities:

- if $n$ is odd, then $C=\{1\}$.

- if $n$ is even, and $n>2$, then $C=\mu_{2} \subset \mu_{n} \subset D_{n}$.

- if $I \cong D_{2} \cong \mathbb{Z}_{2} \times \mathbb{Z}_{2}$, then $C=I$.

Proof. Easy. Left to the reader.

We will now describe the action of $C$ on $\operatorname{Hom}_{\mathrm{Ho}(\mathbf{2} \mathbf{G p})}(\Gamma, \mathrm{PGL}(d, d))_{\chi}$. We assume $I=D_{n}, n$ even. For a non-trivial element $a \in C$, define the map $a^{*}: \Gamma \rightarrow \mathbb{Z}_{2}$ to be the composite $q \circ \chi$, where $q: D_{n} \rightarrow \mathbb{Z}_{2}$ is the map obtained by killing $\mu_{n} \subset D_{n}$, when $n>2$. When $n=2, q$ is $D \rightarrow D / a=\mathbb{Z}_{2}$.

Lemma 9.12. Setup being as above, conjugation by $a \in C$ sends a triple $(\iota, E, \rho)$ in $\operatorname{Hom}_{\mathrm{Ho}(\mathbf{2} \mathbf{G p})}(\Gamma, \operatorname{PGL}(d, d))_{\chi}$ to the triple $\left(\iota, E, \rho^{\prime}\right)$, where $\rho^{\prime}=\rho a^{*}$ (pointwise product). Here, we have used the same notation $a^{*}$ for the precomposition of $a^{*}$ with the projection map $E \rightarrow \Gamma$, and we have also identified $\mathbb{Z}_{2}$ with the corresponding scalar matrices.

Proof. We may assume that $I=D_{n}=\mu_{n} \rtimes \mathbb{Z}_{2}$ is the standard Dihedral group at the origin, with $n$ even. Here we have identified $\mu_{n}$ with the group of matrices

$$
\left(\begin{array}{cc}
\xi & 0 \\
0 & 1
\end{array}\right) \in \mathrm{PGL}_{2}
$$

and the generator of $\mathbb{Z}_{2}$ is the matrix

$$
\left(\begin{array}{cc}
0 & 1 \\
1 & 0
\end{array}\right) \in \mathrm{PGL}_{2}
$$

First we analyze the conjugation action of $a$ on $J$, where $J \in \mathrm{GL}_{2}$ is the inverse image of $I$. This is a central $\mathbb{C}^{*}$ extension of $I$. We only do the case $n>2$; the case $n=2$ is similar.

In this case the centralizer of $I$ is $\mu_{2}$. We assume $a$ is non-trivial and choose the lift $\tilde{a}$ of $a$ to be the matrix

$$
\left(\begin{array}{cc}
-1 & 0 \\
0 & 1
\end{array}\right) \in \mathrm{GL}_{2}
$$

It is easy to see that the conjugation action of this matrix leaves any matrix in $J$ that lies above $\mu_{n} \subset I$ invariant, whereas it sends any other matrix $M$ in $J$ to $-M$; to see this, it is enough to take $M$ to be the following matrix:

$$
\left(\begin{array}{ll}
0 & 1 \\
1 & 0
\end{array}\right)
$$

Now, $\rho$ factors through $J$, so it follows from the above description that the conjugation action of $a$ sends $\rho$ to $\rho a^{*}$. This proves the assertion.

We can think of $a^{*}$ as a character of $\Gamma$; that is, $a^{*} \in \Gamma^{*}$, where $\Gamma^{*}$ stands for the character group of $\Gamma$. This construction actually gives us an injection of $C$ in $\Gamma^{*}$ which sends $a$ to $a^{*}$. We denote the image by $C^{*} \subseteq \Gamma^{*}$. This is a subgroup of order 1,2 or 4 .

Proposition 9.13. In Lemma 9.12, $(\iota, E, \rho)$ and $\left(\iota, E, \rho^{\prime}\right)$ are isomorphic, if and only if $a^{*}$ is in the image of the $d^{\text {th }}$ power homomorphism $\Gamma^{*} \rightarrow \Gamma^{*}$. 
Proof. First assume $a^{*}=\theta^{d}$, for some character $\theta: \Gamma \rightarrow \mathbb{C}^{*}$. Define $f: E \rightarrow E$ by $f(x)=x \iota(\theta(\bar{x}))$, where $\bar{x}$ denotes the image of $x$ in $\Gamma$ under the projection map $E \rightarrow \Gamma$. It is obvious that $f$ is an extension automorphism. It also satisfies $\rho^{\prime}=\rho \circ f$, where $\rho^{\prime}:=c_{a} \circ \rho$ is the map described in Lemma 9.12. This implies that the triples $(\iota, E, \rho)$ and $\left(\iota, E, \rho^{\prime}\right)$ are isomorphic.

Conversely, assume $(\iota, E, \rho)$ and $\left(\iota, E, \rho^{\prime}\right)$ are isomorphic, and let the isomorphism be induced by some $f: E \rightarrow E$. By definition, $f$ commutes with $\iota$ and the projection map to $\Gamma$, and it satisfies the relation $\rho^{\prime}=\rho \circ f$. For an element $x \in E$ define $\theta(x)=x^{-1} f(x)$. This is an element in the image of $\iota$, so it gives us a map $\theta: E \rightarrow \mathbb{C}^{*}$. In fact, since $\iota(\mathbb{C})$ is a central subgroup of $E$, it follows that $\theta$ is a group homomorphism. The relation $\rho^{\prime}=\rho \circ f$ implies that $a^{*}=\theta^{d}$.

Corollary 9.14. Let $D=C^{*} / C^{*} \cap\left(\Gamma^{*}\right)^{d}$. Each orbit of $\operatorname{Hom}_{\mathrm{Ho}(\mathbf{2} \mathbf{G p})}(\Gamma, \mathrm{PGL}(d, d))_{\chi}$ under conjugation action is a set of order $|D|$.

Proof. This follows immediately from Proposition 9.13.

Remark that $|D|=1,2$ or 4 . Except when $n$ and $d$ are both even, we have $|D|=1$, and $|D|=4$ can only happen when $n=2$.

Summarizing the above discussion, if the image $I$ of the homomorphism $\chi: \Gamma \rightarrow$ $\mathrm{PGL}_{2}$ is not a dihedral group, then any two elements in $\operatorname{Hom}_{\mathrm{Ho}(\mathbf{2 G p})}(\Gamma, \mathrm{PGL}(d, d))_{\chi}$ that are conjugate are indeed equal. If $I$ is dihedral, the conjugacy classes have size 1, 2 or 4 (the first case always happens if at least one of $d$ or $n$ is odd, the last case can only happen when $I=D_{2}$ ). To determine which is the case, one has to compute the characters $a^{*}: \Gamma \rightarrow \mathbb{Z}_{2}$ as described above and determine which ones are $d^{t h}$ power. The ones that are $d^{t h}$ power induce trivial conjugation action on $\operatorname{Hom}_{\mathrm{Ho}(\mathbf{2} \mathbf{G p})}(\Gamma, \operatorname{PGL}(d, d))_{\chi}$.

\section{Appendix: 2-Groups}

In this appendix we gather some results on 2-groups and crossed-modules that are used throughout the paper. Our main reference is [8]. More can be found in [4] (and references therein) and [1].

Quick recall on 2-groups. A 2-group $\mathfrak{G}$ is a group object in the category of groupoids. Alternatively, we can define a 2 -group to be a groupoid object in the category of groups, or also, as a (strict) 2-category with one object in which all 1-morphisms and 2-morphisms are invertible (in the strict sense). If we require that the 1-morphisms are only equivalences and not necessarily strictly invertible, what we obtain is called a weak 2 -group. ${ }^{6} 7$

Example. Self-equivalences of an object $X$ in a 2-category (say a stack in the 2category of stacks) naturally form a weak 2 -group Aut $(X)$.

Remark 10.1. Weak 2-groups $\mathfrak{G}$ can be strictified in a functorial way ([8]), and strictification retains all the (homotopical) properties of the original weak 2-group. For this reason in practice we will only need to work with strict 2-groups.

\footnotetext{
${ }^{6}$ In the case that we are interested in (namely, automorphisms of stacks) associativity holds strictly, so we have not weakened associativity in our definition of a weak 2-group. That is why we are sometimes sloppy and drop the adjective weak.

${ }^{7}$ Fortunately, we do not have to worry about the general strictification machinery for weak 2 -groups, because the only weak 2 -group we encounter indeed comes with a natural strictification (Theorem 8.1).
} 
A morphism $f: \mathfrak{G} \rightarrow \mathfrak{H}$ of 2 -groups is a map of groupoids that respects the group operation on the nose. If we view $\mathfrak{G}$ and $\mathfrak{H}$ as 2-categories with one object, such $f$ is nothing but a strict 2-functor. Same definition applies to weak 2-groups (because we have not weakened associativity).

To a 2-group $\mathfrak{G}$ we associate the groups $\pi_{1} \mathfrak{G}$ and $\pi_{2} \mathfrak{G}$ as follows. The group $\pi_{1} \mathfrak{G}$ is the set of isomorphism classes of object of the groupoid $\mathfrak{G}$. The group structure on $\pi_{1} \mathfrak{G}$ is induces from the group operation of $\mathfrak{G}$. The group $\pi_{2} \mathfrak{G}$ is the group of automorphisms of the identity object $e \in \mathfrak{G}$. This is an abelian group. Any morphism $f: \mathfrak{G} \rightarrow \mathfrak{H}$ of (weak) 2-groups induces homomorphisms on $\pi_{1}$ and $\pi_{2}$. We say such $f$ is an equivalence if both these maps are isomorphisms. (Warning: an equivalence need not have an inverse. Also, two equivalent 2-groups may not be related by an equivalence, but just a zig-zag of equivalences.)

We are usually interested in 2-groups up to equivalence, so we will think of a 2 -group as an object in the homotopy category of 2-groups. This category is defined by taking the category of 2-groups and inverting the equivalences. For the reader's information, we point out that, there is a model structure on the category of 2groupoids (see for instance [12]); 2-groups are the pointed connected objects in this category.

Quick recall on crossed-modules. A crossed-module $\mathfrak{G}=\left[\varphi: G_{2} \rightarrow G_{1}\right]$ is a pair of groups $G_{1}, G_{2}$, a group homomorphism $\varphi: G_{2} \rightarrow G_{1}$, and a (right) action of $G_{1}$ on $G_{2}$, denoted ${ }^{-g}$, that lifts the conjugation action of $G_{1}$ on the image of $\varphi$ and descends the conjugation action of $G_{2}$ on itself. The kernel of $\varphi$ is a central (in particular abelian) subgroup of $G_{2}$ and is denoted by $\pi_{2} \mathfrak{G}$. The image of $\varphi$ is a normal subgroup of $G_{1}$ whose cokernel is denoted by $\pi_{1} \mathfrak{G}$. A (strict) morphism of crossed-modules is a pair of group homomorphisms which commute with the $\varphi$ maps and respect the action of $G_{1}$ on $G_{2}$. A morphism is called an equivalence if it induces an isomorphism on $\pi_{1}$ and $\pi_{2}$.

Equivalence of 2-groups and crossed-modules There is a natural pair of inverse equivalences of categories between the category $\mathbf{2 G p}$ of 2 -groups and the category CrossedMod of crossed-modules. Furthermore, these functors preserve $\pi_{1}$ and $\pi_{2}$. Here is how these functors are defined.

Functor from 2-groups to crossed-modules. Let $\mathfrak{G}$ be a 2 -group. Let $G_{1}$ be the group of objects of $\mathfrak{G}$, and let $G_{2}$ be the set of all arrows emanating from the identity object $e ; G_{2}$ is also a group (namely, it is a subgroup of the group of arrows of $\mathfrak{G}$ ).

Define $\varphi: G_{2} \rightarrow G_{1}$ by $\varphi(\alpha):=t(\alpha), \alpha \in G_{2}$.

The action of $G_{1}$ on $G_{2}$ is given by conjugation. That is, given $\alpha \in G_{2}$ and $g \in G_{1}$, the action is given by $g^{-1} \alpha g$.

Functor from crossed-modules to 2-groups. Let $\left[\varphi: G_{2} \rightarrow G_{1}\right]$ be a crossed-module. Consider the groupoid $\mathfrak{G}$ whose underlying set of objects is $G_{1}$ and whose set of arrows is $G_{1} \times G_{2}$. The source and target maps are given by $s(g, \alpha)=g$, $t(g, \alpha)=g \varphi(\alpha)$.

Two arrows $(g, \alpha)$ and $(h, \beta)$ such that $g \varphi(\alpha)=h$ are composed to $(g, \alpha \beta)$.

The group operation on the set of objects $\mathrm{Ob} \mathfrak{G}=G_{1}$ is naturally extended to a group operation on $\mathfrak{G}$ by setting $(g, \alpha)(h, \beta)=\left(g h,\left(\alpha^{h}\right) \beta\right)$, where $-{ }^{h}$ stands for the action of $G_{1}$ on $G_{2}$. 
Remark 10.2. With the above equivalence of categories in mind, we use the words 2 -group and crossed-module synonymously throughout the paper. Every discrete group $\Gamma$ gives rise to a 2 -group in the obvious way. The crossed-module presentation for this group is $[1 \rightarrow \Gamma]$. This gives a full embedding of the category of groups in the category of 2 -groups (or crossed-modules).

10.1. Morphisms into 2-groups. Let $\Gamma$ be a discrete group and $\mathfrak{G}$ a 2 -group. We are interested in determining the homotopy classes of morphisms from $\Gamma$ to $\mathfrak{G}$. This arises in the study of group actions on stacks. Namely, let $\mathcal{X}$ be a stack, and $\mathfrak{G}=\operatorname{Aut}(X)$ be the automorphism 2-group of $X$ (rather, its strictification). Then the actions of $\Gamma$ on $\mathcal{X}$ (up to a reasonable notation of equivalence) are in a natural bijection with homotopy classes of maps from $\Gamma$ to $\mathfrak{G}$.

Caveat. Strictly speaking, our use of the expression 'homotopy classes of morphisms from $\Gamma$ to $\mathfrak{G}$ ' is not quite correct. What we really mean is 'elements in $\operatorname{Hom}_{\mathrm{Ho}(\mathbf{2} \mathbf{G p})}(\Gamma, \mathfrak{G})^{\prime}$. The point is that, not every element in $\operatorname{Hom}_{\mathrm{Ho}(\mathbf{2} \mathbf{G p})}(\Gamma, \mathfrak{G})$ can be realized by a strict morphism of 2 -groups $\Gamma \rightarrow \mathfrak{G}$, but only a weak one.

The following result is proved in [8].

Theorem 10.3 ([8], Corollary 6.9). Let $\mathfrak{G}$ be a 2-group and let $\left[\varphi: G_{2} \rightarrow G_{1}\right]$ be the crossed-module presentation for it. Let $\Gamma$ be a discrete group. Then, the set $\operatorname{Hom}_{\mathrm{Ho}(\mathbf{2} \mathbf{G p})}(\Gamma, \mathfrak{G})$ of homotopy classes of morphisms from $\Gamma$ to $\mathfrak{G}$ is in a natural bijection with the set of isomorphism classes of commutative diagrams of the form

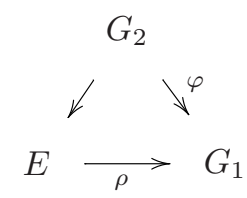

where $E$ is an extension of $\Gamma$ by $G_{2}$, and for every $x \in E$ and $g \in G_{2}$ we require that $g^{\rho(x)}=x^{-1} g x$. An isomorphism between two such diagrams $(E, \rho)$ and $\left(E^{\prime}, \rho^{\prime}\right)$ is, by definition, an isomorphism of extensions $f: E^{\prime} \rightarrow E$ (so it induces identity on $G_{2}$ and $\Gamma$ ) such that $\rho^{\prime}=\rho \circ f$.

In terms of actions of discrete groups on stacks, this can be interpreted as follows. Let $X$ be a stack (or, more generally, an object in a 2-category). Take $\mathfrak{G}$ to be a strict model for the (weak) 2-group of automorphisms of $\mathcal{X}$, with $\left[\varphi: G_{2} \rightarrow G_{1}\right]$ the crossed-module presentation for it. The elements of $G_{1}$ are then to be thought of as a complete collection of (2-isomorphism classes) of automorphisms of $\mathcal{X}$, while the elements of $G_{2}$ parameterize 2-isomorphisms between them. Of course, too few actions of $\Gamma$ on $X$ can be actually obtained as group homomorphisms $\Gamma \rightarrow G_{1}$, as we have to allow certain "laxness". What the theorem is saying is that, there is, however, a canonical way of weaving the elements of $G_{2}$ into $\Gamma$ to form a larger group $E$ so that our action now can be realized as an actual group homomorphism $E \rightarrow G_{1}$, with $G_{2} \subseteq E$ keeping track of the 2-isomorphisms.

For computational purposes, it is useful to have the cohomological versions of Theorem 10.3 handy.

Theorem 10.4 ([8], Proposition 7.6). Let $\mathfrak{G}$ be a 2-group, and let $\Gamma$ be a discrete group. Fix a homomorphism $\chi: \Gamma \rightarrow \pi_{1} \mathfrak{G}$. Consider the set $\operatorname{Hom}_{\mathrm{Ho}(\mathbf{2} \mathbf{G p})}(\Gamma, \mathfrak{G})_{\chi}$ of 
homotopy classes of maps $\Gamma \rightarrow \mathfrak{G}$ inducing $\chi$ on $\pi_{1}$. Then, either this set is empty, or it admits a natural transitive action of $H^{2}\left(\Gamma, \pi_{2} \mathfrak{G}\right)$. (Here, $\pi_{2} \mathfrak{G}$ is made into a $\Gamma$-module via $\chi$.)

Definition 10.5. We say a 2 -group $\mathfrak{G}=\left[G_{2} \rightarrow G_{1}\right]$ is split if the projection map $G_{1} \rightarrow \pi_{1} \mathfrak{G}$ admits a section. (Note: this definition is slightly different from the one used in [8].)

Corollary 10.6. In Theorem 10.3, assume the 2-group $\mathfrak{G}$ is split, and fix a section $\sigma: \pi_{1} \mathfrak{G} \rightarrow G_{1}$. Then, there is a natural bijection

$$
\operatorname{Hom}_{\mathrm{Ho}(\mathbf{2} \mathbf{G p})}(\Gamma, \mathfrak{G})_{\chi} \longleftrightarrow H^{2}\left(\Gamma, \pi_{2} \mathfrak{G}\right) .
$$

In Theorem 10.3, the action of $H^{2}\left(\Gamma, \pi_{2} \mathfrak{G}\right)$ can be made completely explicit (see $[8]$ ), and this allows us to write down every homotopy class $\Gamma \rightarrow \mathfrak{G}$, provided that we are given one of them to begin with. We will show how to do this in the special case where $\mathfrak{G}$ is split. First we need to introduce a notation.

Definition 10.7. Let $H, G$ and $K$ be groups, each equipped with a right action of $K$, the one on $K$ itself being conjugation. We denote the actions by $-{ }^{k}$. Assume we are given a $K$-equivariant diagram:

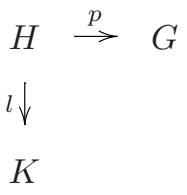

satisfying the compatibility condition $g^{l(h)}=p(h)^{-1} g p(h)$, for $h \in H$ and $g \in G$. The semi-direct product $K \ltimes_{H} G$ of $K$ and $G$ along $H$ is defined to be $K \ltimes G / N$, where

$$
N=\left\{\left(l(h)^{-1}, p(h)\right), h \in H\right\} .
$$

Corollary 10.8 ([8], Corollary 7.9). Suppose the natural projection $G_{1} \rightarrow \pi_{1} \mathfrak{G}$ has a section, and fix such a section $\sigma: \pi_{1} \mathfrak{G} \rightarrow G_{1}$. Then, every homotopy class $\Gamma \rightarrow \mathfrak{G}$ is uniquely characterized by a pair $(K, \chi)$, where $\chi: \Gamma \rightarrow \pi_{1} \mathfrak{G}$ is a group homomorphism and $K$ is (the isomorphism class of) an extension of $\Gamma$ with $\pi_{2} \mathfrak{G}$ :

$$
1 \rightarrow \pi_{2} \mathfrak{G} \rightarrow K \rightarrow \Gamma \rightarrow 1 \text {. }
$$

Given such an extension we obtain a diagram as in Theorem 10.3 by setting $E:=$ $K \ltimes_{\pi_{2} \mathfrak{G}} G_{2}$ and $\rho(k, \alpha):=\sigma(\chi(\bar{k})) \varphi(\alpha),(k, \alpha) \in E$. Here, the action of $K$ on $G_{2}$ is obtained via $\sigma \circ \chi$ from that of $G_{1}$ on $G_{2}$. The notation $\bar{k}$ stands for the image of $k \in K$ in $\Gamma$.

Again, we can interpret this result in terms of group action on stack as follows. First of all, to fix a section $\sigma: \pi_{1} \mathfrak{G} \rightarrow G_{1}$ means to fix a strict action of $\pi_{1} \mathfrak{G}$ on $X$. The corollary now says that, to give an action of $\Gamma$ on $\mathcal{X}$ is the same as letting an extension $K$ of $\Gamma$ by $\pi_{2} \mathfrak{G}$ act strictly on $\mathcal{X}$, with $\pi_{2} \mathfrak{G} \subseteq K$ acting trivially.

10.2. Conjugations in 2-groups and crossed-modules. Given a 2-group $\mathfrak{G}$ and an element $a$ in $G_{1}$ (the "group of objects") we define the automorphism, $c_{a}: \mathfrak{G} \rightarrow$ $\mathfrak{G}$, called conjugation by $a$, to be the map that sends an object $h$ (respectively, and arrow $\alpha$ ) to $a^{-1} h a$ (respectively, $a^{-1} \alpha a$ ). This is obviously a map of 2 -groups. On 
the crossed-module $\left[\varphi: G_{2} \rightarrow G_{1}\right]$ associated to $\mathfrak{G}$, the conjugation by $a$ acts as conjugation by $a$ on $G_{1}$ and as $-{ }^{a}$ on $G_{2}$.

Using this we can define the notion of conjugacy classes of maps between 2groups. This notion passes to the homotopy category. In particular, we can also talk about conjugacy classes of maps between two objects in the homotopy category of 2-groups.

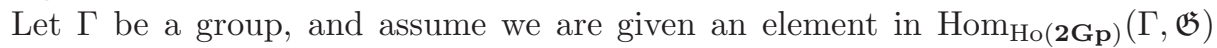
represented by a diagram

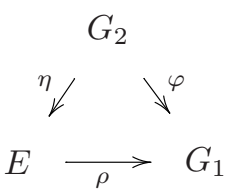

as in Theorem 10.3. For $a \in G_{1}$, the conjugation action $c_{a}: \operatorname{Hom}_{\mathrm{Ho}(\mathbf{2} \mathbf{G p})}(\Gamma, \mathfrak{G}) \rightarrow$ $\operatorname{Hom}_{\mathrm{Ho}(\mathbf{2} \mathbf{G p})}(\Gamma, \mathfrak{G})$ sends this diagram to the diagram

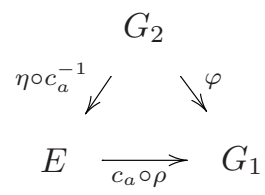

Here we have abused the notation and denoted the conjugation action of $a$ on $G_{1}$, as well as the action of $a$ on $G_{2}$, by $c_{a}$.

Let us examine the effect of the conjugation action from the cohomological point of view. For a given $\chi: \Gamma \rightarrow \pi_{1} \mathfrak{G}$, and an element $a \in G_{1}$, the conjugation $c_{a}: \mathfrak{G} \rightarrow$ $\mathfrak{G}$ induces a natural map

$$
a_{*}: \operatorname{Hom}_{\mathrm{Ho}(\mathbf{2} \mathbf{G p})}(\Gamma, \mathfrak{G})_{\chi} \rightarrow \operatorname{Hom}_{\mathrm{Ho}(\mathbf{2} \mathbf{G p})}(\Gamma, \mathfrak{G})_{c_{\bar{a}} \circ \chi} .
$$

This map is equivariant in the following sense. Recall that $\operatorname{Hom}_{\mathrm{Ho}(\mathbf{2} \mathbf{G p})}(\Gamma, \mathfrak{G})_{\chi}$ is an $H^{2}\left(\Gamma, \pi_{2} \mathfrak{G}\right)$-set (Theorem 10.4). Similarly, $\operatorname{Hom}_{\operatorname{Ho}(\mathbf{2 G p})}(\Gamma, \mathfrak{G})_{c_{\bar{a}} \circ \chi}$ is an $H^{2}\left(\Gamma, \pi_{2} \mathfrak{G}\right)_{a^{-}}$ set. The index ${ }_{a}$ here is to emphasis that, in the latter cohomology group the $\Gamma$-action on $\pi_{2} \mathfrak{G}$ is different from the one in the former cohomology group; the two actions are conjugate by the automorphism $-^{a}: \pi_{2} \mathfrak{G} \rightarrow \pi_{2} \mathfrak{G}$. The automorphisms $-{ }^{a}: \pi_{2} \mathfrak{G} \rightarrow \pi_{2} \mathfrak{G}$, which is $\Gamma$-equivariant for the two actions, induces an isomorphism $\alpha: H^{2}\left(\Gamma, \pi_{2} \mathfrak{G}\right) \rightarrow H^{2}\left(\Gamma, \pi_{2} \mathfrak{G}\right)_{a}$. The map $a_{*}$ is an $\alpha$-equivariant map.

Corollary 10.9. Notation being as above, assume a has the following properties: 1) the action of a on $\pi_{2} \mathfrak{G}$ is trivial, 2) $c_{a} \circ \chi=\chi$ and there is a class in $\operatorname{Hom}_{\mathrm{Ho}(\mathbf{2} \mathbf{G p})}(\Gamma, \mathfrak{G})_{\chi}$ that is invariant under $a_{*}$. Then $a_{*}$ is identity.

Proof. We may assume $\operatorname{Hom}_{\mathrm{Ho}(\mathbf{2 G p})}(\Gamma, \mathfrak{G})_{\chi}$ is non-empty. By hypothesis, the isomorphism $\alpha: H^{2}\left(\Gamma, \pi_{2} \mathfrak{G}\right) \rightarrow H^{2}\left(\Gamma, \pi_{2} \mathfrak{G}\right)_{a}$ is identity. Since $a_{*}$ is an $\alpha$-equivariant self-map of a transitive $H^{2}\left(\Gamma, \pi_{2} \mathfrak{G}\right)$-set, and since it has a fixed point, it must be identity.

\section{REFERENCES}

1. K. Brown, Cohomology of groups, Graduate Texts in Mathematics, 87, Springer-Verlag, New York, 1994.

2. R. Fox, On Fenchel's conjecture about F-groups, Mat. Tidsskr. B. (1952), 61-65. 
3. I Kra H.M. Farkas, Riemann surfaces, Graduate Texts in Mathematics, 71, Springer-Verlag, New York, 1992.

4. A. Lauda J. Baez, Higher dimensional algebra v: 2-groups, arXiv math.QA/0307200.

5. J. Lurie, Tannaka duality for geometric stacks, arXiv math.AG/0412266.

6. B. Maskit, Kleinian groups, Grundlehren der Mathematischen Wissenschaften, 287, SpringerVerlag, Berlin, 1988.

7. B. Noohi, Foundations of topological stacks I, Preprint.

8.

9. (2004).

10. P.E.Schupp R.C. Lyndon, Combinatorial group theory, Ergebnisse der Mathematik und ihrer Grenzgebiete, Band 89, Springer-Verlag, Berlin-New York, 1977.

11. J-P. Serre, Trees, Springer-Verlag, 1980.

12. I. Moerdijk; J. Svensson, Algebraic classification of equivariant homotopy 2-types., J. Pure Appl. Algebra 89 (1993), 187-216. 